\title{
Intrinsic network activity in human brain organoids
}

Tal Sharf, ${ }^{1,2}$ Tjitse van der Molen, ${ }^{1,2}$ Elmer Guzman, ${ }^{1,2}$ Stella M.K. Glasauer, ${ }^{1,2}$ Gabriel Luna, ${ }^{1,2}$ Zhouwei Cheng, ${ }^{4}$ Morgane Audouard, ${ }^{1,2}$ Kamalini G. Ranasinghe, ${ }^{5}$ Kiwamu Kudo, ${ }^{6}$ Srikantan $S$. Nagarajan, ${ }^{6}$ Kenneth R. Tovar, ${ }^{1,2}$ Linda R. Petzold,${ }^{4}$ Paul K. Hansma, ${ }^{1,3}$ Kenneth S. Kosik ${ }^{1,2 *}$

${ }^{1}$ Neuroscience Research Institute, University of California Santa Barbara, Santa Barbara, CA 93106, USA.

${ }^{2}$ Department of Molecular, Cellular and Developmental Biology, University of California Santa Barbara, Santa Barbara, CA 93106, USA

${ }^{3}$ Department of Physics, University of California Santa Barbara, Santa Barbara, CA 93106

${ }^{4}$ Department of Computer Science, University of California Santa Barbara, Santa Barbara, CA 93106, USA

${ }^{5}$ Memory and Aging Center, Department of Neurology, University of California San Francisco, San Francisco, CA 94158, USA

${ }^{6}$ Department Radiology and Biomedical Imaging, University of California San Francisco, San Francisco, CA 94143, USA

*e-mail: kosik@lifesci.ucsb.edu

\begin{abstract}
Human brain organoids replicate much of the cellular diversity and developmental anatomy of the human brain. However, the physiological behavior of neuronal circuits within organoids remains relatively under-explored. With high-density CMOS microelectrode arrays and shank electrodes, we probed broadband and three-dimensional spontaneous activity of human brain
\end{abstract}


organoids. These recordings simultaneously captured local field potentials (LFPs) and single unit activity. From spiking activity, we estimated a directed functional connectivity graph of synchronous neural network activity which showed a large number of weak functional connections enmeshed within a network skeleton of significantly fewer strong connections. Increasing the intrinsic inhibitory tone with a benzodiazepine altered the functional network graph of the organoid by suppressing the network skeleton. Simultaneously examining the spontaneous LFPs and their phase alignment to spiking showed that spike bursts were coherent with theta oscillations in the LFPs. An ensemble of spikes phase-locked to theta frequency oscillations were strongly interconnected as a sub-network within the larger network in which they were embedded. Our results demonstrate that human brain organoids have self-organized neuronal assemblies of sufficient size, cellular orientation, and functional connectivity to coactivate and generate field potentials from their collective transmembrane currents that phaselock to spiking activity. These results point to the potential of brain organoids for the study of neuropsychiatric diseases, drug mechanisms, and the effects of external stimuli upon neuronal networks.

\section{Introduction}

Under a variety of conditions, ${ }^{1} 3 \mathrm{D}$ assemblies of pluripotent stem cells can differentiate into a wide diversity of brain cell types and assume certain anatomical features that resemble the developing brain including some mature features such as dendritic spines, inhibitory and excitatory synapses ${ }^{2-4}$ with presynaptic vesicles. ${ }^{5}$ Neurons within these $3 \mathrm{D}$ assemblies generate action potentials upon depolarization, ${ }^{6}$ display excitatory and inhibitory post synaptic currents ${ }^{7}$ and exhibit spontaneous network activity as measured by calcium imaging ${ }^{6,8,9}$ and low density 
extra-cellular field potential recordings. ${ }^{5,10,11}$ Recent work has extended the activity repertoire of organoids to include rhythmic activity in the delta (1-4 Hz) and gamma frequency (100-400 Hz) ranges. ${ }^{12}$ However, technological limitations have restricted high-bandwidth simultaneous detection of electrical activity to small numbers of neurons. Consequently, technical shortcomings have hindered attempts to determine functional connectivity among large numbers of neurons, how they collectively synchronize with rhythmic activity and how pharmacologic perturbations affect these physiologic parameters. Recent advances in CMOS-based microelectrode array (MEA) technolgies ${ }^{13}$ have enabled large-scale ex vivo mapping of cortical synaptic projections. ${ }^{14}$ We utilize this state-of-the-art technology to generate detailed electrical activity maps from human brain organoids using arrays with 26,400 recording electrodes that tile across $\approx 8.1 \mathrm{~mm}^{2}$ surface area equivalent to the cross-section of our brain organoids. The electrode pitch approximates the size of a single neuron, thereby allowing the assignment of individual units to single neurons rather than the more limited ascertainment of multi-unit activity from the considerably sparser arrays used in previous studies. We utilized a 960 electrode Neuropixels CMOS shank probe, ${ }^{15}$ also with an electrode pitch approximately the size of a neuron soma, to gain access to the z-plane of the organoid. We then constructed a spatial connectivity map which was derived from sub-millisecond temporal resolution of extra-cellular action potentials measured simultaneously across a thousand microelectrodes spatially-routed from the 26,400 total set of electrodes. We detected theta frequency oscillations supported by their coherence across the surface of the organoid and stereotypical sets of neuronal ensembles phase-locked to the theta oscillation. These results enhance the utility of brain organoids for novel approaches to the study of the neuropharmacological underpinning of network behavior, as well as conditions such as schizophrenia, autism and neurodegeneration. 


\section{Results}

High resolution spike map across a human brain organoid. $500 \mu \mathrm{m}$ thick organoid slices were positioned over the recording electrode surface of high-density CMOS MEAs (MaxOne, Maxwell biosystems) and seated with a sterile custom harp slice grid for recording up to six months after placing on the arrays (Suppl. Fig.1, Methods). Slicing of organoids can enhance cell growth, prevent interior cell death ${ }^{11}$ and promote the development of extensive axon outgrowth. ${ }^{10}$ Once positioned on the MEA, the onset of activity varied across organoids, then increased in connectivity and firing rates and later often diminished possibly due to the difficulty of sustaining viability over the long term (Suppl. Fig. 2). A high-density field recording (electrode diameter $7.5 \mu \mathrm{m}$, pitch of $17.5 \mu \mathrm{m}$ corresponding approximately to the size of a single neuronal soma) was obtained from tissue areas of approximately $2.1 \mathrm{~mm}$ by $3.85 \mathrm{~mm}$. A spikeactivity map was derived by performing an automated scan of contiguous tiled blocks selected from 26,400 recording electrode sites using switch-matrix CMOS technology ${ }^{16}$ that enabled configurable routing of up to 1,024 simultaneous recording electrode sites across the surface of the array (Fig. 1a). By recording from the 1,020 top electrodes based on activity we generated detailed spatiotemporal maps of spiking activity, often in the form of synchronized bursts (Fig. 1b), that varied in relative spiking activity across the tissue section (Fig. 1a). To validate that spikes arose from fast synaptic transmission, we blocked AMPA and NMDA receptors with bath application of NBQX $(10 \mu \mathrm{M})$ and R-CPP $(20 \mu \mathrm{M})$ and $\mathrm{GABA}_{\mathrm{A}}$ receptors with Gabazine (10 $\mu \mathrm{M}$ ) resulting in a $72 \% \pm 29 \%$ (mean \pm STD) reduction in spiking (active channels with at least 30 detected spikes over 2 minutes; $n=4$ organoids). The remaining active channels likely reflect action potentials from interneurons firing in the absence of synaptic input. ${ }^{17}$ A saturating concentration of the sodium-channel blocker tetrodotoxin $(1 \mu \mathrm{M})$ was subsequently applied to 
the same organoid slices, resulting in a $98 \% \pm 1 \%$ (mean \pm STD) reduction in spiking activity compared to control conditions (Suppl. Fig. 3e-h), indicating that only a very small fraction of the signals were falsely detected spikes.

To increase the inhibitory tone within organoid circuits we used the benzodiazepine, diazepam, which increases the frequency of $\mathrm{GABA}_{\mathrm{A}}$ receptor-mediated inhibitory postsynaptic potentials. ${ }^{18}$ Treatment with diazepam at a concentration of $50 \mu \mathrm{M}$ decreased the inter-burst intervals, burst duration and the number of units participating in the burst (burst amplitude) throughout the organoid ( $n=4$ organoids, Fig. 1b and Suppl. Fig. 3a-d for diazepam dose responses and additional statistics), consistent with diazepam effects in neocortical slice cultures over the same concentration windows. ${ }^{19}$ By increasing the conductances of inhibitory circuits we observed $\mathrm{a} \approx 40 \%$ reduction in the average burst duration.

To determine that spiking activity extended in the z-plane of the organoids, as opposed to the $2 \mathrm{D}$ recordings from slices on 2D CMOS arrays, we performed acute electrophysiology recordings from intact brain organoids with a Neuropixels probe, a densely tiled 960 electrode CMOS shank with $20 \mu \mathrm{m}$ inter-electrode pitch. ${ }^{15}$ Acute electrical recordings were performed by inserting the CMOS shank into the organoid using a motorized, low-drift micromanipulator. We observed single-unit spiking activity that extended hundreds of microns across the organoid tissue within a limited stratum of the z-plane for $n=3$ organoids (Fig. 2a,b). Similar to the results from organoid slices, we observed spontaneous, synchronized population bursts.

We characterized the cellular composition of the organoids to validate the presence of cell types consistent with the observed physiological features. Organoids often have a relatively nutrient deficient central region (Fig. 3a) likely due to diffusion limits of the media that cause loss of cell viability, which may account for the peripheral ring of higher spiking activity (Fig. 1a 
and Suppl. Fig. 4 for spiking activity from additional organoids). Whole-sections from 8-month old organoids demonstrated the presence of stellate astrocytes well-connected by gap junction proteins (Fig.3a). Axons coursed through dendritic fields that appeared directionally aligned in some regions and can project over long distances (Fig. 3b). Various interneuron types were present including parvalbumin-labeled cells (Fig. 3c), ${ }^{7,20}$ as well as synaptobrevin decorated MAP2-positive dendrites (Fig. 3d). To obtain additional detail about the cellular composition of the organoids we performed single-cell sequencing on three 7-month-old brain organoids. Unsupervised clustering and dimensionality reduction resulted in the separation of nine major cell populations consisting of cortical excitatory neurons, GABAergic interneurons, astrocytes, an additional VIM+ glial cluster, oligodendrocyte progenitors, and a small cluster of choroid plexus cells (Fig. 3e and Suppl. Fig. 5). GABAergic cells expressed both GAD1 and GAD2 (Suppl. Fig. 6) as well as several DLX genes indicating their origin in the ventral forebrain (Suppl. Fig. 6). Within both neuronal clusters (glutamatergic and GABAergic), several subunits of GABA receptors associated with network oscillations ${ }^{21}$ and AMPA and NMDA receptor genes were expresses (Suppl. Fig.6). The forebrain marker FOXG1 was expressed in all these major cell populations. In contrast, markers for other brain regions (retina, midbrain and hindbrain) were only minimally expressed (Suppl. Fig.6), indicating predominant forebrain identity of our organoids. $^{22}$

These results and related observations in the literature ${ }^{2-8,10-12}$ suggest that self-assembled brain organoids possess the basic architectural motifs necessary to form functional neuronal circuitry in a 3-dimensional space. The innate circuitry has the capacity to form complex, synaptically coupled electrogenic networks, composed of principal neurons and GABAergic interneurons which are critically involved in driving network oscillations. ${ }^{21}$ Below, we quantify 
the precise spatiotemporal signatures of spikes and extra-cellular field potentials that arise from endogenous activity in our brain organoids.

Spike Time Correlation Map. To construct a functional connectivity map based on the spiking activity (Fig. 1a), a correlation measure for any pair of spike trains was defined by the spike time tiling coefficient (STTC). ${ }^{23}$ The STTC measure depends on a single adjustable parameter $(\Delta t)$, indicating a time window around spikes. This results in a value ranging between 1 (strong correlation) and -1 (strong anti-correlation). Our pipeline first removed redundant, high fidelity spikes that represent action potential propagation along single axons, known as propagation signals. ${ }^{24}$ Our organoids $(n=6)$ at 7 months had a $14 \pm 4 \%$ (mean \pm STD) channel redundancy. Redundant signals of this type have been observed on high density arrays and can resolve the spatial extent of the soma and axonal arborisation by tracking action potentials along processes spanning millimeter length scales in $2 \mathrm{D}$ dissociated cultures. ${ }^{25} \mathrm{We}$ observed redundant signal length scales of $35 \mu \mathrm{m} \pm 20 \mu \mathrm{m}$ (mean $\pm \mathrm{STD}, n=6$ organoids) across the CMOS recording surface (Suppl. Fig.7), which are limited in their length detection due to the extension of axonal arbors into the z-plane.

Using this filtered data set, a spike synchronicity window $(\Delta t)$ of $20 \mathrm{~ms}$ was used to compute the functional connectivity scores for each pair of electrodes ${ }^{23}$ to generate a weighted functional connectivity network based on single unit activity (Methods, Fig. 1c and Suppl. Fig 8,9). The relative delay times between correlated spike trains and spike-time jitter resulted in a mean latency within $\approx 5$ ms time window and very few longer than $10 \mathrm{~ms}$ (Fig.1d and Suppl. Fig. 9i). These results suggested functional coupling between neurons within the organoid. ${ }^{26}$ Predominant directionality was assigned to the edges of the functional connectivity map based 
on the spike latency distributions between electrode pairs (see Methods for additional details).

Briefly, unimodal spike latency distributions (Hartigans' dip test $P>0.1$ ) with non-zero mean latencies (greater than axonal conduction timescales ${ }^{24,25}$ over the mm length scale of our organoids) were considered significant and consistent with the unidirectional nature and timescales of synaptic transmission. ${ }^{26-29}$ A negative mean spike time latency compared to the reference channel indicated a predominant directionality towards the reference channel and vice versa (Fig. 1d). Bidirectional connections as suggested by distinctly bimodal latency distributions were excluded from the analysis (Suppl. Fig. 9e,g,h). Based on the directionality, the in-degree $\left(D_{\text {in }}\right)$ and out-degree $\left(D_{\text {out }}\right)$ were computed per channel, defined as the number of incoming and outgoing edges respectively. Nodes with a high fraction of outgoing edges $\left(D_{\text {out }}-\right.$ $\left.D_{\text {in }}\right) /\left(D_{\text {out }}+D_{\text {in }}\right)>0.8$ were labelled 'sender' nodes (Fig. 1f, top). Nodes with a high fraction of incoming edges $\left(D_{\text {in }}-D_{\text {out }}\right) /\left(D_{\text {out }}+D_{\text {in }}\right)>0.8$ were labelled 'receiver' nodes (Fig. 1f, middle). Differences in the directionality vector less than 0.8 were labelled 'broker' nodes, which represented the majority of the nodes (senders: $20 \% \pm 9.2 \%$, brokers: $56 \% \pm 7.7 \%$, receivers: $24 \% \pm 5.6 \%$ (mean \pm STD)). These data were computed from four independent organoids (Suppl. Fig. 10a).

The spike time correlation connectivity map revealed a traffic pattern through the organoid visualized as a graph of interconnected nodes (Fig. 1e). The largest component—a graph of contiguous interconnected nodes-was parameterized according to its connection strengths, number of nodes and their degree. Setting the lower limit of connectivity strength at 0.35 minimized spurious connections (see Methods for randomization details and Suppl. Fig. 2f). Edge strengths and node degree did not fit a random distribution (Fig. 4a, Suppl. Fig. 11). Rather, both a truncated power law $^{30}$ and a Lorentzian ${ }^{31}$ fit these distributions well, with the 
truncated power law out-performing the Lorentzian for the edge strength distributions in most cases and vice versa for the node degree distributions (Methods and Suppl. Fig. 11). This result was consistent with observations that describe connectivity in cortical networks as a "skeleton of strong connections among a sea of weaker ones." 32,33

Diazepam $(50 \mu \mathrm{M})$ decreased the number of spikes in each burst (Fig. 1b and Suppl. Fig. 3a,b), decreased the number of nodes on the connectivity map (Fig. 1g) mostly by decreasing the number of receiver nodes and reduced the size of the largest component of connected nodes (Suppl. Fig. 10c). This observation suggested that diazepam reduced the number of states or active functional relationships that the organoid explores. At a stringent connectivity strength threshold, increasing the intrinsic inhibition with diazepam almost completely eliminated the skeleton of strong connections, while increasing the sea of weaker connections (Fig. 4).

Theta frequency oscillations. To extract periodic oscillatory activity from the extra-cellular field, we decomposed the broad-band voltage vs. time signals into local field potential (LFP) frequency sub-bands by band-pass filtering raw data (Fig.5a, see Methods). We limited this analysis to theta oscillations (4-8 Hz) (Suppl. Fig.12-14) considered significant over time intervals when the oscillation amplitude envelope exceeded the noise floor. Multiple brief theta cycles occur at different spatial locations across the organoid (Fig. 5b, red lines) that can be referenced to burst activity (Fig.5b, black line) signified when the population-averaged spike rate peaks within the burst. Within a narrow time window these theta oscillations show consistent phase offsets relative to each other (Fig. 5c, gray lines). When we blocked AMPA, NMDA and $\mathrm{GABA}_{\mathrm{A}}$ receptors along with sodium channels, the measured LFP amplitudes were no longer 
observable above the CMOS amplifier noise floor ( $n=4$ organoids; Suppl. Fig. 13). This

validates that the LFP signals depended upon synaptic transmission-based network activity. ${ }^{34}$

Cross-correlation coefficients of theta oscillations and their relative time-lags between all electrode pairs were used to generate a spatial correlation map with a focal region of highest correlated activity and their phase delays relative to more distant positions on the map (Fig. 5d). Signal averaging theta oscillations with respect to oscillation peaks above the noise floor from the highest correlated node (identified in Fig. 5d) further validated synchronized theta activity with relative phase shifts across the organoid (Fig. 5e,f), see Suppl. Fig. 15 for visualizations in a different organoid. We further demonstrated that LFPs were synchronized with neuronal population bursts (see next section for further details and the work of Buzsaki et al. ${ }^{34}$ ). Next, we confirmed the validity of the LFP theta signal by estimating imaginary coherence, a metric which is dependent on non-zero phase synchrony ${ }^{35}$ and found sets of highly coherent electrodes (Suppl. Fig. 16) which corresponded to the same spatial regions identified by cross-correlation and signal averaged analysis (Fig. 5d-i). The benzodiazepine diazepam induced a dramatic reduction in pairwise theta correlations (Suppl. Fig. 17d) and significant decrease in the global imaginary coherence ( $n=3$ organoids, paired t-test, $P<1 \mathrm{e}-4$, Suppl. Fig. 16f).

Peak theta amplitudes are synchronized with neuronal population bursts. The amplitude of theta oscillations increased when synchronized with neuronal population bursts (Suppl. Fig. 14), an effect that has been observed in vivo. ${ }^{34,36}$ First, we identified the time points when the neuronal population firing rates (averaged over a $5 \mathrm{~ms}$ time window) peaked within burst epochs. Burst-peak times were then used as temporal anchor points to signal average theta oscillations (Fig. 5g,h) across all electrode sites. A spatial map (Fig. 5i) of signal-averaged theta oscillations 
(relative to the neuronal population burst-peak) revealed regions of theta activity strongly correlated with burst peaks. Signal averaging theta amplitudes relative to population burst-peaks revealed the same spatial pattern of theta phase coherence attained by independently signal averaging theta oscillations relative to their peak oscillation amplitude time points (Fig. 5e,f). Theta phase alignment was maximized within a $100 \mathrm{~ms}$ time window relative to the population burst peak (red dotted line Fig. 5g and inset) and shown by a sharp drop in the circular standard deviation of the phase. Temporal windows of constant phase shifts (defined where the circular standard deviation of the theta phase dropped below one) persisted over a $\approx 400 \mathrm{~ms}$ time window or roughly 2-3 theta cycles. The time spent above the noise floor substantially increased during broader burst periods ( $n=4$ organoids, Suppl. Fig.14). Modulating GABA A $_{\text {conductances with }}$ diazepam $(50 \mu \mathrm{M})$ induced a notable reduction $(>30 \%)$ in the number of correlated theta electrode sites (above a correlation threshold of 0.2) across the organoids $(n=3)$ (Suppl. Fig. 17d), a result consistent with the known down-regulation of theta activity by benzodiazepines in vivo. ${ }^{37,38}$

To confirm synchronization of theta oscillations to neuronal population bursts we performed acute, high-bandwidth extra-cellular field potential recordings from whole organoids using a Neuropixels shank $(n=3)$. We next identified electrode sites along the z-plane of the organoid that exhibited prominent theta band oscillations in the LFP (Fig.2c). To determine if the population bursts were phase-synchronized to theta oscillations in the intact organoids, we signal-averaged theta phases relative to the population burst peak times along the shank (Fig. 2d,e). Theta phase alignment was synchronized with population bursts and co-localized with spatial regions where spiking activity was observed (Fig. 2e, right). Meanwhile theta phases added deconstructively in regions where the organoid showed no signs of spiking activity. Our 
high-bandwidth measurements of electrical activity generated by brain organoids provided strong evidence that theta is synchronized over temporal epochs of increased neuronal activity within the time window of population bursts. ${ }^{34}$

Spike phase-locking to theta. Endogenous brain activity can entrain action potentials to theta frequency oscillations ${ }^{39}$ and define a temporal window for spiking within local circuits. ${ }^{40,41}$ Previous work in vivo and ex vivo has relied on manually positioned, low channel count recording electrodes, to identify a handful of units exhibiting preferential spike phase-locking to theta frequencies at a given moment in time. ${ }^{39,40,42,43}$ We looked for relationships between theta frequency oscillations and action potential spikes in organoids using the high density and large spatial extent of the CMOS arrays. To determine whether spikes occurred at a preferred theta phase, we counted spikes in relation to the phase of theta cycles (across 1,020 electrodes) (Fig. 6a) and determined significance with the Rayleigh criterion for non-uniform distribution of spikes in circular phase space. ${ }^{39}$ With the spatial-routing of a thousand microelectrodes (out of 26,400), we simultaneously surveyed both theta oscillations and spikes simultaneously across the organoid to draw a spatial map of the mean phase-locked spike angle to theta for regions of highly correlated theta activity identified previously by the cross-correlation analysis (Fig. 6c and Suppl. Fig. 18 for analysis from $n=3$ organoids). Theta-phase spike event distributions for electrode location revealed a strong preference for spiking during a narrow phase window of theta centered about its mean angle $\mu$ (Fig. 6b). Finally, utilizing acute high-bandwidth field recordings from whole brain organoids using a Neuropixels shank we confirmed the presence phase-locking of spikes to theta (satisfying the Rayleigh criterion) (Fig. 2f) simultaneously 
across multiple sites (Fig. 2g) in the z-plane of the organoid, a result and was consistent across $n$ $=3$ whole organoids.

We next sought to quantify the connectivity among the population of sites across the organoid that were phase-locked to theta oscillations. We found that phase-locked sites form an interconnected ensemble composed of higher relative connectivity strengths when compared against the larger constituent connectivity network in which they reside (Fig. 7a,b), significance between these two connectivity distributions was determined using a KS-test $(P<4 \mathrm{e}-28)$. To further show the shift in connectivity strengths did not represent spurious subset of the larger connectivity network, we compared the connectivity strengths from phase-locked ensemble against randomly drawn edges within the largest connectivity component, yielding a significant difference between the distributions ( $n=5$ randomly drawn networks, $P<4 \mathrm{e}-14)$. Higher mean connectivity weights were consistently observed across $n=3$ organoids (Fig.7c and Suppl. Fig 19). These findings suggested that intrinsic fields at theta frequencies, which in the brain can provide a temporal window for computation within local circuits, ${ }^{40}$ may play a role in defining tightly coupled spike ensembles which serve to reinforce strong connections relative to the very large number of weaker connections.

\section{Discussion}

The demonstration of activity networks in brain organoids further enhances their utility as a model of human brain function. The robust detection of signals was facilitated by the use of high-density CMOS microelectrode arrays that can monitor spiking with high temporal and spatial resolution in relation to theta oscillations. The organoids were prepared according to undirected methods ${ }^{8}$ which consist predominantly of forebrain, while midbrain and hindbrain cellular populations can arise within the same organoid. When cultured for prolonged time 
periods organoids can undergo substantial neuronal maturation, including the elaboration of dendritic spines and the formation of spontaneously active neuronal networks. ${ }^{5}$ Indeed, our single cell analysis and IHC demonstrated the cellular diversity potentially capable of supporting LFPs (Fig. 3 and Suppl. Fig. 5,6). For example, a variety of inhibitory neurons were present, including parvalbumin cells (Fig. 3c) which are known to be associated with theta oscillations ${ }^{44}$ and axon tracts that extended over millimeter ranges (Fig. 3b). Single-cell transcriptomics indicate the presence of functioning cells capable of forming complex neural networks including the presence of GABAergic cells that are consistent with their role in the generation of highly correlated activity networks detected as LFPs. ${ }^{45}$

The formation of a connectivity map (Fig.1e) demonstrated that the organoid was capable of establishing a weighted network of inflow and outflow tracts over short and long distances. As demonstrated in the rat brain, ${ }^{32,33}$ high connection strength edges shape a non-random framework against a background of weaker ones (Fig.1e, Suppl. Fig.11e-g). Node degree which corresponds to single units (Suppl. Fig. 8), also displayed a relatively small number of highly connected nodes and a higher abundance of less connected nodes (Suppl. Fig 11a-d). The majority of the nodes, which we refer to as brokers, have large proportions of both incoming and outgoing edges. This short duration snapshot could over longer time scales calibrate a dynamic balance among receivers and senders as a global mechanism of plasticity. Increasing the intrinsic effects of inhibition with diazepam reduced the variation in node degree and selectively weakened strong connections relative to the sea of weaker connections (Fig. 4 and Supp. Fig. 11), thereby suggesting that organoid networks are capable of instantiating multiple states.

While the theoretic basis of LFPs is understood, ${ }^{46}$ the detailed structural geometries of current sources, especially dendritic processing of synaptic inputs, ${ }^{47-49}$ can vary greatly. We 
found that cerebral organoids—composed of roughly one million cells—have neuronal assemblies of sufficient size, cellular orientation, connectivity and co-activation capable of generating field potentials in the extra-cellular space from their collective transmembrane currents. The measurements from the organoid are conducted within a small volume of tissue $(\approx$ $3.5 \mathrm{~mm}^{3}$ ), which greatly diminishes the problems of volume conduction from distant different sources.

The correlations between the theta oscillations and local neuronal firing strongly supported a local source for the rhythmic activity. ${ }^{34}$ Consistent with minimal volume effects, we validated theta oscillations by demonstrating that the imaginary part of coherency ${ }^{35}$ explained the entirety of the coherency. The local volume through which theta dispersed extended to the zdimension as shown with the Neuropixels shank (Fig. 2). Finally, we show that phase-locked neurons form a tightly interconnected ensemble, which is composed of the strongly weighted connectivity network edges (Fig. 7). These findings open up the possibility that endogenous fields within brain organoids may serve as an experimental system for enhancing the function and timing within local circuits as observed in vivo. ${ }^{40}$

Activity, as observed here, in the absence of an external stimulus fits what O'Keefe and Nadel referred to in describing the cognitive map as a Kantian synthetic a priori system $^{50}$ manifest as a physiologic scaffold capable of representing information from the external environment before it is experienced. In fact, spontaneous synchronization of local neuronal populations with LFPs persist in the absence of sensory and motor outputs occur in vivo ${ }^{36}$ and carry information about the underlying state of the neuronal network. ${ }^{51}$ In practical terms, the theta-frequency phase-locking resembling that observed in humans ${ }^{52}$ suggest that brain organoids 
can serve as an excellent platform for the investigation of drug effects and disease effects on brain network activity.

\section{Acknowledgements}

This study was funded by the Arnold O. Beckman Postdoctoral Fellowship Award (T.S.), Dr.

Miriam and Sheldon G. Adelson Medical Research Foundation (K.S.K.), National Institutes of Health grant number K08AG058749 (K.G.R.), R01NS100440 (S.S.N.), R01AG062196 (S.S.N.), UCOP-MRP-17-454755 (S.S.N.), Larry L. Hillblom Foundation: 2019-A-013-SUP (K.G.R.), Alzheimer Nederland (T.V.D.M.), Swiss National Science Foundation Early Postdoc Mobility and Postdoc Mobility grants P2ZHP3-174753 and P400PB-186800 (S.M.K.G.).

\section{Author contributions}

T.S. and K.S.K. designed, conceived and supervised the study; P.K.H. and L.R.P. offered numerous suggestions and comments. T.S. designed and performed electrophysiology experiments on sliced organoids. T.S., T.V.D.M. and E.G. performed computational analysis and statistics on electrophysiology recordings. E.G. performed shank recording on whole organoids; S.M.K.G. cultured the organoids, performed single-cell RNA sequencing and analysis; G.L. performed immunohistochemistry, imaging and organoid slicing; K.G.R. and K.K. performed imaginary coherence analysis under the supervision of S.S.N.; Z.C. performed additional analyses.; M.A. contributed to cell culturing; K.R.T. supervised pharmacology experiments; T.S. and K.S.K wrote the manuscript, and all authors discussed the results and commented on the manuscript. 


\section{Additional information}

Competing interests. The authors declare that they have no competing interests.

\section{Methods}

Cerebral organoid generation. The control iPCS line F12442.4 ${ }^{53}$ was cultured in mTeSR1 medium (Stem Cell Technologies) on tissue culture plates coated with hESC-qualified Matrigel (Corning). mTeSR1 was exchanged every other day and iPSCs were routinely passed using ReLeSR (Stem Cell Technologies). Cerebral organoids were generated after the method by Lancaster et $a l .{ }^{8}$ with minor modifications. iPSCs were incubated in $0.5 \mathrm{mM}$ EDTA in D-PBS for 3 min before dissociation in Accutase for 3 min at $37^{\circ} \mathrm{C}$. After adding mTeSR 1 and triturating to achieve a single-cell suspension, cells were pelleted for $3 \mathrm{~min}$ at 1,000 rpm. Cells were resuspended in low bFGF hES media (20\% KOSR, 3\% ES-FBS, 1x GlutaMAX, 1x MEMNEAA, 1x $\beta$-Mercaptoethanol, 4 ng/ml bFGF in DMEM/F12) supplemented with Rock inhibitor $(50 \mu \mathrm{M})$ and plated in U-bottom ultra-low attachment plates at 4,500 cells per well. On day 2, low bFGS hES media with Rock inhibitor was replaced. Another media change was performed on day 4, with omission of bFGF and Rock inhibitor. On day 5, embryoid bodies were transferred to neural induction media (1x N2 supplement, 1x GlutaMAX, 1x MEM-NEAA, 1ug/ml Heparin in DMEM/F12), and. media was replaced on days 7 and 9. On day 10, each neuroepithelial structure was embedded in 15 ul hESC-qualified Matrigel, followed by incubation in neural induction media for another 2 days. On day 12, neural induction media was replaced with NeuroDMEM-A media (0.5x N2 supplement, 1x B27supplement without Vitamin A, 1x $\beta$-Mercaptoethanol, 1x GlutaMAX, 0.5x MEM-NEAA, 250 ul/1 insulin solution, 1x Pen/Strep in 50\% DMEM/F12 and 50\% Neurobasal). From day 19 on, organoids were cultured 
in NeuroDMEM+A media (0.5x N2 supplement, 1x B27 supplement with Vitamin A, 1x $\beta$ -

Mercaptoethanol, 1x GlutaMAX, 0.5x MEM-NEAA, 250 ul/1 insulin solution, 12.5 mM HEPES, $0.4 \mathrm{mM}$ Vitamin C, 1x Pen/Strep in 50\% DMEM/F12 and 50\% Neurobasal) with media changes twice per week. From day 21 on, organoids were kept on an orbital shaker at $75 \mathrm{rpm}$.

Mycoplasma testing was routinely performed on organoids and iPSCs using the MycoAlert Mycoplasma DetectionKit (Lonza).

Immunohistochemistry and microscopy. Organoid samples were fixed in $4 \%$ paraformaldehyde in $0.1 \mathrm{M}$ sodium cacodylate buffer (pH:7.4) overnight at $4{ }^{\circ} \mathrm{C}$. After rinsing, samples were embedded $10 \%$ agarose and sectioned $(100 \mu \mathrm{m})$ using a vibratome (Leica, Lumberton, NJ). Primary antibodies used were anti-Parvalbumin (1:200; abcam, Cambridge, MA; ab11427), anti-GAD65 (1:200; GeneTex, Irvine, CA; GTX113192), anti-SMI312 (1:500; BioLegend, San Diego, CA; 801701), anti-GFAP (1:500; abcam, Cambridge, MA; ab53554), anti-connexin 43 (1:200; Millipore, Burlington, MA; MAB3067), anti-MAP2 (1:500; GeneTex, Irvine, CA; GTX82661), anti-synaptobrevin (1:500; Synaptic Systems, Goettingen, Germany; 104-211). Samples were viewed through a 20x oil immersion lens (N.A. 0.85) and imaged using an upright Olympus Fluoview 1000 laser scanning confocal microscope (Center Valley, PA) equipped with a motorized stage. High-resolution wide-field mosaics were produced as described elsewhere. ${ }^{54}$

\section{Single-cell RNA sequencing of cerebral organoids}

Organoid dissociation. Individual cerebral organoids were subjected to dissociation using the Worthington Papain Dissociation System (Worthington). Solutions were prepared according to 
the Manufacturer's instructions. Organoids in NeuroDMEM+A (defined in Cerebral organoid generation) media were cut into pieces with a sterile scalpel blade and washed twice in PBS prior to dissociation. Incubation in papain/DNaseI solution was performed at $37^{\circ} \mathrm{C}$ and $5 \% \mathrm{CO}_{2}$ with occasional agitation. After the first 30 minutes of incubation, organoids were triturated 10 times with a fire-polished glass Pasteur pipette, followed by further incubation and trituration every 15 minutes until dissociation was complete. The total incubation time in papain/DNaseI solution was 90 min. After adding Inhibitor Solution and DNaseI in EBSS, the dissociated cells were pelleted by centrifugation for 3 minutes at $300 \mathrm{~g}$. The cells were resuspended in $500 \mu \mathrm{l}$ ice-cold PBS/0.01\% BSA using a wide-bore plastic pipette tip and passed through a $35 \mu \mathrm{m}$ cell strainer. An aliquot of the single-cell suspension was mixed with Trypan blue and analyzed for viability and cell concentration on a Countess II FL Automated Cell Counter (ThermoFisher). Viability was $>90 \%$ for all organoid samples.

Drop-seq library preparation. Following dissociation, cells were diluted to 110 cells/ $\mu 1$ in PBS/0.01\% BSA. Drop-seq was performed as described in Macosko et al. ${ }^{55}$ Briefly cells and barcoded beads (Chemgenes, 132 beads/ul in lysis buffer) were run on an aquapel-treated microfluidic drop-seq device (FlowJEM) for co-encapsulation in nanoliter-sized droplets. After droplet-breakage, reverse transcription and Exonuclease I treatment, beads were counted, and 2,500 beads were apportioned per PCR tube for cDNA amplification. The amplified cDNA libraries were purified using SPRI beads (Beckman Coulter) and quantified on a Fragment Analyzer (Agilent). Tagmentations were performed using the Illumina Nextera XT kit (Illumina), and the resulting libraries were purified in two consecutive rounds of SPRI beadsbased size selection ( $0.6 \mathrm{x}$ beads to sample ratio followed by $1 \mathrm{x}$ beads to sample ratio). The size 
and concentration of the final libraries were measured on a Fragment Analyzer and a Qubit Fluorometer (ThermoFisher), respectively. Libraries were sequenced on an Illumina Nextseq500 instrument.

Drop-seq data analysis. Counts matrices were generated using the Drop-seq tools package. ${ }^{55}$ Briefly, raw reads were converted to BAM files, cell barcodes and UMIs were extracted, and low-quality reads were removed. Adapter sequences and polyA tails were trimmed, and reads were converted to Fastq for STAR alignment (STAR version 2.6). Mapping to the human genome (hg19 build) was performed with default settings. Reads mapped to exons were kept and tagged with gene names, beads synthesis errors were corrected, and a digital gene expression matrix was extracted from the aligned library. We extracted data from twice as many cell barcodes as the number of cells targeted (NUM_CORE_BARCODES $=2 \mathrm{x} \#$ targeted cells). Downstream analysis was performed using Seurat $3.0^{56,57}$ in $\mathrm{R}$ version 3.6.3. An individual Seurat object was generated for each sample, and objects were merged using the Seurat merge function. Cells with $<300$ genes detected were filtered out, as were cells with $>10 \%$ mitochondrial gene content. Counts data were log-normalized using the default NormalizeData function and the default scale of 1e4. The top 3,000 variable genes were identified using the Seurat FindVariableFeatures function (election.method = "vst", nfeatures $=3,000$ ), followed by scaling and centering using the default ScaleData function. Principal Components Analysis was carried out on the scaled expression values of the 3,000 top variable genes, and the cells were clustered using the first 30 principal components (PCs) as input in the FindNeighbors function, and a resolution of 0.4 in the FindClusters function. Non-linear dimensionality reduction was performed by running UMAP on the first 30 PCs. Following clustering and dimensionality 
reduction, putative cell doublets were identified using DoubletFinder, ${ }^{58}$ assuming a doublet formation rate of $5 \%$ and calculating homotypic proportions based on clustering at resolution 0.4 as described above. A pk value of 0.61 was selected for doublet identification based on the results of paramSweep_vs, summarizeSweep and find.pK functions of the DoubletFinder package. After filtering out the doublets as identified by DoubletFinder, counts data were extracted, and a new Seurat object was generated. Normalization, variable gene selection, scaling, clustering and UMAP dimensionality reduction were performed as described above with the exception that 32 PCs were used as input for clustering and UMAP. Clustering was performed at different resolutions $(0.3,0.4,0.6$ and 0.8). Clustering at resolution 0.4 resulted in 9 clusters and was in good agreement with the expression of known marker genes for cell types found in brain organoids (Suppl. Fig. 5b). This resolution was therefore selected for delineating different cell populations. Marker genes were identified for each cluster using the Wilcoxon rank sum test implemented the FindMarkers function, with a $\log \mathrm{FC}$ threshold of 0.25 and Bonferroni correction for multiple testing, confirming and refining our initial cell type annotation using canonical marker genes.

\section{Interfacing cerebral organoid sections with high-density CMOS microelectrode arrays.}

Whole cerebral organoids were allowed to mature an age of 4-6 months old (see Cerebral organoid generation section) and were then embedded in $10 \%(\mathrm{w} / \mathrm{v})$ low melting point agarose and allowed to cool at $2{ }^{\circ} \mathrm{C}$ for $\approx 10$ minutes. Organoids embedded in the agarose gel were then sectioned into $500 \mu \mathrm{m}$ thick slices with a vibratome (Leica VT1000S) using an advance speed of one and a vibration speed of nine. The gel block containing the organoid was mounted onto the surface of the vibratome cutting block with super glue and placed into the cutting reservoir filled 
with ice-cold cutting solution composed of $50 \%$ (v/v) DMEM (ThermoFisher), $50 \%$ (v/v) Neurobasal (ThermoFisher), 1x penicillin streptomycin (ThermoFisher). The space surrounding the vibratome liquid reservoir was filled with ice to keep the cutting solution temperature constant during sectioning. The vibratome blade was allowed to advance through the front-face of the gel block and organoid tissue, while leaving $\approx 3 \mathrm{~mm}$ gel uncut and attached at the back of the gel block. This method allowed previous sections to remain attached to the gel block, which minimized torque on the organoid during subsequent sections and prevented deforming or releasing of the remaining uncut tissue embedded in the gel below. Next, the contiguous layer stack of $500 \mu \mathrm{m}$ thick organoid sections remaining in the gel block (Suppl. Fig. 1a) were then peeled off using sterile forceps (in a dissection hood equipped with a stereoscopic microscope) and placed into individual wells in a 6 well plate filled with cutting solution using a cut p1000 pipette tip. The slices were then transferred to individual wells in a 12 well plate filled with BrainPhys neuronal medium (StemCell Technologies) with the addition of the following supplements 2\% (v/v) NeuroCultTM SM1 Neuronal Supplement (StemCell Technologies), 1\% (v/v) N2 Supplement-A (StemCell Technologies), 20 ng/ml Recombinant Human Brain-Derived Neurotrophic Factor (StemCell Technologies), 20 ng/ml Recombinant Human Glial-Derived Neurotrophic Factor (StemCell Technologies), 1 mM Dibutyryl-cAMP (PeproTech), 200 nM ascorbic acid (StemCell Technologies), 1x penicillin streptomycin (ThermoFisher) and allowed to recover for 24 hours in a $5 \% \mathrm{CO}_{2}$ incubator $37^{\circ} \mathrm{C}$ prior to placement and positioning on the surface of CMOS MEAs.

High density CMOS MEAs and custom machined (UCSB physics machine shop) liquid reservoir lids made from Delrin (McMaster-Carr), fitted with $\mathrm{CO}_{2}$ permeable, water vapor 
impermeable membranes, ${ }^{59}$ were first sterilized in a $70 \%(\mathrm{v} / \mathrm{v})$ ethanol solution in deionized water (18.2 M $\Omega-\mathrm{cm})$ for 30 minutes. The liquid reservoir of the CMOS MEA was then rinsed thoroughly with sterile ultra-pure distilled water in a sterile hood $(\approx 5 \mathrm{ml}$ per CMOS MEA well) and the lids were allowed to air dry in the sterile hood. The recording surface of the MEAs were then coated by adding $0.5 \mathrm{ml}$ of poly-1-lysine (PLL) (Sigma Aldrich) solution $(0.1 \mathrm{mg} / \mathrm{ml}$ in ultra-pure water) into the CMOS MEA reservoirs and then placed in an incubator for 30 minutes at $37{ }^{\circ} \mathrm{C}$ with the lids on. The arrays were then transferred to a sterile hood, the PLL solution was then aspirated off and washed an additional 3x with ultra-pure water ( $1 \mathrm{ml}$ volume for each wash). The CMOS MEA reservoir was then filled with $0.5 \mathrm{ml}$ of BrainPhys medium and the lid was placed on it. The CMOS MEA was then transferred to a sterile dissection hood equipped with a stereoscopic microscope. Organoid sections were then transferred to the CMOS MEA well with a cut P1000 pipette tip and gently positioned over the recording electrode surface of the CMOS MEA with sterile forceps while visualizing with the stereoscopic microscope (Suppl. Fig. 1b). A sterile custom harp slice grid was used to seat the organoid slice to the CMOS MEA surface. The harp slice grid was made by attaching $122 \mu \mathrm{m}$ nylon fibers (taken from plastic mesh 9318T45, McMaster-Carr) spaced at a $1 \mathrm{~mm}$ pitch to a stainless-steel washer (M3 lock washer, McMaster-Carr) with epoxy (Devcon). The organoid sections on top of the CMOS MEAs were then maintained in an incubator $\left(5 \% \mathrm{CO}_{2}\right.$ at $\left.37^{\circ} \mathrm{C}\right)$ and media was exchanged twice a week.

Electrophysiology recordings. High density extracellular field potential recordings were measured in a cell culture incubator $\left(5 \% \mathrm{CO}_{2}\right.$ at $\left.37^{\circ} \mathrm{C}\right)$ using complementary metal-oxidesemiconductor (CMOS) micro-electrode array technology (MaxOne, Maxwell biosystems) containing 26,400 recording electrodes with an inter-electrode pitch of $17.5 \mu \mathrm{m}$, an electrode 
size of $7.5 \mu \mathrm{m}$, and a sensing area of $3.85 \mathrm{~mm}$ x $2.1 \mathrm{~mm}$, of which 1,024 can be selected for simultaneous recording. ${ }^{60}$ The low noise amplifiers have a high pass filter $(0.5 \mathrm{~Hz})$ to minimize drift. Electrical measurements were performed once a week (24 hours after a media change) and took around two weeks after sectioning to start spiking with a significant increase in activity after 1 month (Suppl. Fig. 2) on the array. Automatic activity scans (tiled blocks of 1,020 electrodes) were performed to identify the spatial distribution of electrical activity across the surface of the organoid. Data was sampled at $20 \mathrm{kHz}$ for all recordings and saved as HDF5 file format.

We chose the routed top most spiking 1,020 electrodes to have a minimum spacing distance of at least two electrodes $(2 \times 17.5 \mu \mathrm{m})$, which allowed optimal positioning of the recording electrode to the soma diameter. Because the amplitude of the extra-cellular action potential falls off inversely with distance, ${ }^{34,61}$ and electrode spikes are determined by amplitudes that exceeded $4 \mathrm{x}$ the rms-noise of each electrode chanel, ${ }^{62}$ this programmable electrode routing gave us primarily single unit activity as demonstrated (Fig. 1c, Suppl. Fig.8).

MEA signal processing. Raw signals (sampled at $20 \mathrm{kHz}$ ) were imported into MATLAB (Mathworks) for analysis. The data was filtered into high frequency spiking and low frequency local field potential (LFP) components. The spiking component was extracted by- band-pass filtering (cutoff frequencies of 0.3 and $7 \mathrm{kHz}$ using a $4^{\text {th }}$ order Butterworth filter) the raw voltage signal and spike detection was set at $4 \mathrm{x}$ the rms-noise for a given electrode channel. ${ }^{62}$ Detected spike times (rounded to the nearest $\mathrm{ms}$ ) were then stored as a binary spike time matrix (columns represent electrodes and rows represent time). The LFP component was extracted by first low pass filtering the raw data (frequency cutoff of $500 \mathrm{~Hz}$ using $4^{\text {th }}$ order Butterworth filter), and 
then down sampled to $1 \mathrm{kHz}$. The LFP was then filtered into the traditional oscillatory sub-bands using a bi-directional finite impulse response (FIR) filter. ${ }^{63,64}$ Delta $(0.5-4 \mathrm{~Hz})$, theta $(4-8 \mathrm{~Hz})$, alpha $(8-13 \mathrm{~Hz})$, beta $(13-30 \mathrm{~Hz})$ and gamma $(30-50 \mathrm{~Hz})$ bands were extracted using eefilt.m function from the UCSD EEGlab MATLAB toolbox (Suppl. Fig. 12).

\section{Acute recordings from cerebral organoids using a high-density Neuropixels CMOS shank.}

6 month old cerebral organoids were transferred to BrainPhys media and were cultured for 30 days without shaking before recording. Organoids were then positioned using a cut p1000 pipette tip into a custom well (filled with BrainPhys media) to immobilize the organoid (kept at $37{ }^{\circ} \mathrm{C}$ on a temperature controlled stage). A high-density CMOS shank with 960 electrode sites that tile along a 70 x 20 um cross-section shank (10 mm long) where 384 channels can be programmably routed and recorded from simultaneously ${ }^{15}$ was inserted into the organoid using a low drift $(<10$ $\mathrm{nm} / \mathrm{hr}$ ), motorized precision micromanipulator (MP-285, Sutter Instruments). The Neuropixels probe was mechanically attached to the micromanipulator using a custom machined bracket (UCSB physics machine shop). Electrophysiology data was acquired using SpikeGLX (Bill Karsh, https://github.com/billkarsh/SpikeGLX). Action potential channel was sampled at $30 \mathrm{kHz}$ and band pass filtered from 0.2- $4 \mathrm{k} \mathrm{Hz}$. The local field (LF) channel (sampled at 2,500 Hz) was low pass filtered at $500 \mathrm{~Hz}$. Downstream processing and analysis was performed with methods similar to 2D CMOS data.

Population-averaged spike rate and population bursts. Population-averaged spike rates were calculated by first summing all detected spikes (across all 1,020 channels) occurring within a 1 ms time window to create a population spike vector. The population spike vector was then 
averaged over a $20 \mathrm{~ms}$ sliding window and smoothed with a $100 \mathrm{~ms}$ Gaussian kernel, yielding a population-averaged spike rate as measured across the CMOS array. Next, population bursts peaks were defined when the population-averaged spike rate exceeded $2 \mathrm{x}$ its rms value (using the findpeaks function with a minimum peak distance of $1 \mathrm{~s}$ ). Population burst durations were defined by time windows surrounding the burst peak where the burst amplitude was attenuated by $90 \%$ of its maximum value. To calculate the fine burst structure details, a 5 ms sliding window average used, followed by a $20 \mathrm{~ms}$ Gaussian smoothing kernel.

Multimodal waveform distributions. To assess the fraction of electrodes that records activity from more than one neuron, the modality of the waveform amplitude distributions was analysed per electrode. Modality was assessed using Hartigans' dip test for unimodality, ${ }^{65}$ where $P<0.1$ indicates a multimodal distribution. A Monte Carlo simulation was performed to assess the fraction of multimodal distributions that are being classified as multimodal by the Hartigans' dip test $(P<0.1)$. Waveform amplitude values were drawn from two separate normal distributions with standard deviations ranging from 1 to 4 . The difference between the means of the two distributions ranged from 1 to $30 \mu \mathrm{V}$ (stepsize $1 \mu \mathrm{V}$ ). For each voltage difference, 10,000 simulations were performed. For each simulation, a total of 30 and 100 'spike amplitudes' were drawn, with an equal number from each distribution (Suppl. Fig. 8e).

\section{Spike time tiling coefficient to draw a functional connectivity map. Correlated spiking} activity between electrodes was compared using the spike-time tiling coefficient (STTC). ${ }^{23} \mathrm{~A}$ publicly available MATLAB script ${ }^{10}$ was used to compute the STTC. A synchronicity window $(\Delta t)$ of 20 ms was used to compute a score of functional connectivity for each pair of electrodes. 
Electrode pairs that had at least one electrode with less than 30 detected spikes over a $120 \mathrm{~s}$ were removed. To remove propagation signals, the STTC was computed between all electrode pairs for a $\Delta t$ of $2 \mathrm{~ms}$. Channel pairs with an STTC value higher than 0.7 for this time window were considered to reflect propagation signals (see Suppl. Fig. 7 for additional details). ${ }^{24}$ These redundant channels were removed from the channels between which a propagation signal was measured. Firstly, redundant channels with a multimodal distribution (Hartigans' dip test $P<$ 0.1) in the spike waveform amplitudes were identified (Suppl. Fig. 9). Next, among redundant electrode clusters, individual electrodes with the largest average spike amplitudes were selected and all other redundant channels were removed. The remaining correlations between channel pairs were further filtered based on the spike time latency distributions of the spike trains. All electrode pairs with a multimodal latency distribution (Hartigans' dip test $P<0.1$ ), a full width at half maximum (FWHM) larger than $15 \mathrm{~ms}$ or a mean spike time latency between $[-2 ; 2]$ ms were removed. To rule out the influence of outliers, these measures were computed over all spike time latencies within a $[-20 ; 20]$ ms range. Using the STTC scores of the remaining channel pairs, functional connectivity networks were generated.

Lower bound connectivity score estimation. To obtain a lower bound for connectivity scores to use for generating connectivity networks, STTC connectivity scores for randomized spike time vectors were computed. Randomization was performed by selecting the spike times within an individual burst event and shuffling the corresponding electrode numbers. This was done for each burst event individually. This resulted in randomized spike time vectors while maintaining the same population rate as the original recordings. Connectivity scores between electrode pairs were computed in the same way as the original data, including the removal of propagation 
signals and the exclusion of correlations based on the modality, FWHM and mean of the spike time latency distributions (Suppl. Fig. 9). Based on the distributions of the STTC scores of the remaining connections, a lower bound threshold of STTC $=0.35$ was chosen. Within the randomized data sets ( $n=6$ organoids), $97.3 \% \pm 4.6 \%$ percent of the STTC connectivity edges strengths were below the 0.35 threshold across 14 separate measurements (Suppl. Fig. 2f for randomization distributions).

Global connectivity network metrics. Global and local connectivity metrics for the connectivity networks were analysed using the MIT strategic engineering network analysis toolbox for MATLAB. ${ }^{66}$ For networks generated using an STTC, all edges were removed that fell below a threshold of 0.35 (see Lower bound connectivity score estimation for details in Methods). The size of the largest and second largest component, defined as the number of nodes present in the largest and second largest structure of connected nodes. The size of the second largest component was less than $2 \%$ of the size of the largest component for L1, L2, L3 and L4 and less than $10 \%$ for L5 and L6 for 7 month old organoids.

Directional connectivity map. Based on the mean of the spike time latency distributions, directionality was assigned to the edges in the functional connectivity map. A negative mean spike time latency compared to the reference channel indicated a directionality towards the reference channel and vice versa. Based on the directionality, the in-degree $\left(D_{\text {in }}\right)$ and out-degree ( $\left.D_{\text {out }}\right)$ were computed per channel, defined as the number of incoming and outgoing edges respectively. 'Receiver' nodes were defined as $\left(D_{\text {in }}-D_{\text {out }}\right) /\left(D_{\text {in }}+D_{\text {out }}\right)>0.8$, while 'sender' nodes were defined as $\left(D_{\text {out }}-D_{\text {in }}\right) /\left(D_{\text {in }}+D_{\text {out }}\right)>0.8$. All other nodes were labelled brokers. The 
directionality per node was calculated for the largest component of interconnected nodes. To evaluate the significance of sender and receiver nodes, networks were randomized by performing double edge swaps (10 times as many swaps were performed as edges in the control network) and contained the same number of nodes and the same edge density. Randomized networks contained no sender or receiver nodes (Suppl. Fig. 10a).

Diazepam treatment and analysis. Diazepam (Sigma) was solubilized in DMSO (Sigma) at 1,000 times the target concentration and $1 \mu \mathrm{L}$ was then added to the $1 \mathrm{ml}$ CMOS MEA reservoir containing culture media and gently mixed with a p100 pipette. The cultures $(n=4)$ were then allowed to sit undisturbed for $15 \mathrm{~min}$ in the incubator $\left(37^{\circ} \mathrm{C} 5 \% \mathrm{CO}_{2}\right)$ before recording. Organoid dose responses were carried out over 3,10, 30 and $50 \mu \mathrm{M}$ diazepam concentrations (Suppl. Fig. 3a-d).

For connectivity networks generated using recordings from organoids treated with $50 \mu \mathrm{M}$ diazepam, the size of the largest component was compared to the largest component size of networks for control conditions. The shape of the node degree distributions in the largest component then were compared. A power law fit $\left(\mathrm{y}=\mathrm{a} \cdot x^{\mathrm{b}}\right)$, an exponential fit $\left(y=\mathrm{a} \cdot \mathrm{e}^{-\mathrm{c} * x}+\mathrm{d}\right)$ a truncated power law fit $\left(y=\mathrm{a} \cdot x^{\mathrm{b}} \cdot \mathrm{e}^{-\mathrm{c} \cdot x}\right)$ and a Lorentzian fit $\left(\mathrm{y}=\mathrm{a} \cdot 1 /(\gamma * \pi) *\left(\gamma^{2} /\left(\left(x-n_{0}\right)^{2}+\right.\right.\right.$ $\left.\gamma^{2}\right)$ ) ) were performed on the binned node degree distribution (bin size $=3$ ). The goodness of the fit was assessed using the Akaike information criterion (AIC). ${ }^{30,67}$ The width of the node degree distribution, resulting from the Lorentzian fit, was compared to assess changes in the degree distribution of the network after treatment with diazepam. Similarly, a truncated power law fit 
was performed on the binned edge strength distribution (bin size $=0.05$ ) of the edges in the largest component of the network (Suppl. Fig. 11).

The edges that were present in the largest component during both the control and diazepam recording were selected. For each edge, the strength during both treatments was compared. Edges with the highest STTC score for control were pooled together and edges with the highest STTC score for diazepam were pooled together. The weakening of the individual edges in each of the pools was expressed by the percent change in the STTC score compared to the other treatment. The distributions of the percent changes were then plotted as a function of the STTC score under the strongest condition. These distributions were binned (10 bins for STTC ranging from 0.35 until 1. Seven bins for percent changes ranging from 0 until - 60) and the relative fraction of the total number of edges in each bin was computed. The bin values for the pool of edges with the highest STTC score under diazepam conditions were then subtracted from the bin values for the pool of edges with the highest STTC score under control conditions in order to visualize the differences between the two treatments.

Time course in culture analysis. Electrical recordings were made from the organoids $(n=3)$ at 6, 7 and 8 months old. First, population-averaged firing rate (defined above), was calculated for each burst period. The results were averaged over all bursts within a recording, centered about the population burst peak. Secondly, the peak population rate was averaged over all bursts within a recording and compared between organoids at different ages. The average inter-burst interval, defined as the average time between burst peaks was compared as well. Functional connectivity maps based on the STTC were generated for the organoids at different ages. Finally, the size of the network, described by the number of nodes in the largest component were compared. 
Theta coherence. Normalized cross-correlation analysis was performed between all pairwise electrodes $(1,020)$ for the theta band time series using the $x$ corr function in MATLAB. The correlation coefficient and respective lag-time were stored in 1,020 x 1,020 matrices. Before performing pairwise correlations, theta oscillation amplitudes smaller than $10 \mu \mathrm{V}$ were set to $\mathrm{NaN}$ values and considered below the noise floor of the CMOS detectors at this bandwidth (Suppl. Fig. 13 for noise floor measurements with synaptic blockers and TTX). Next, each electrode correlation strength was determined by ranking by the summed correlation coefficient between all pairwise channels. The top correlated channel ('seed' electrode) was then used to generate a spatial correlation and phase-lag map between the other 1,019 channels across the array. This analysis was also repeated for theta time series occurring during population burst periods (see population-averaged firing rate and population bursts in Methods for details). Next, the total number of pairwise theta correlations occurring within the burst periods and during the whole time series were compared. Finally, the total number of pairwise correlations with a correlation score of at least 0.2 was compared between control and diazepam conditions during burst time periods.

To further validate the cross-correlation calculations, theta oscillations were signal averaged relative to the 'seed' electrode. First, positive peaks were identified using the findpeaks function with a minimum peak height of $10 \mu \mathrm{V}$ (see Suppl. Fig 13e and Blockers and TTX analysis in Methods) and a minimum peak distance of $100 \mathrm{~ms}$. Next, the theta peak time points from the 'seed' electrode were used as anchor points to average theta signals centered over a 500 ms time window for each electrode. The amplitude and relative phase shifts (relative to the 
'seed' electrode) were used to generate spatial coherence maps of theta oscillations across the organoid.

Imaginary coherence estimation. The raw LFP signal band pass filtered into the theta band was used to compute the degree of coherence using the imaginary part of the coherence estimation. The imaginary coherence metric between each of the electrode pairs ${ }^{35}$ was computed using a window function with the length of $0.5 \mathrm{~s}$ and $25 \%$ overlap. The connectivity strength at each electrode, regional imaginary coherence, was estimated by averaging across all Fisher's Ztransformed imaginary coherence values. ${ }^{68}$ After generating the imaginary coherence matrix for the full $1,020 \times 1,020$ electrode pairs, we thresholded this data-matrix at the $90^{\text {th }}$ percentile of the imaginary coherence values. We selected the nodes with $>200$ connections in this limited matrix, which resulted in seven highly coherent electrodes (Suppl. Fig. 16b). These seven electrodes were connected to 595 other electrodes in total. These nodes were clustered based on squared Euclidean distance and k-means clustering method (evalclusters function in MATLAB). The clustering algorithm with its highest stability, identified two clusters, and separated a small cluster with 54 electrodes, which was locally distributed on the array (cluster 1; Suppl. Fig. 16c) and had tighter within coherence (Suppl. Fig. 16d). In three different organoids, for each of the 1,020 electrodes, the average strength of the imaginary coherence was calculated as the rowaverages for the $1,020 \times 1,020$ matrix, before and after treatment with diazepam. Paired t-tests comparing the pre- and post-diazepam conditions for each of the organoids showed a significant reduction of imaginary coherence with diazepam treatment (Suppl. Fig. 16f). 
Blocker and TTX experiments and analysis. Preparation of stock solutions to block components of fast synaptic transmission: The AMPA receptor blocker NBQX (Abcam) was solubilized in DMSO, the NMDA receptor blocker R-CPP (Abcam) and the GABA receptor blocker Gabazine (Abcam) were prepared in ultrapure distilled water (Life Tech) at 1,000x the desired working concentration. The working concentrations were 10, 20 and $10 \mu \mathrm{M}$ for NBQX, R-CPP and Gabazine respectively. The sodium channel blocker tetrodotoxin (TTX) citrate (Abcam) was solubilized in ultrapure water at 1,000x the working concentration $(1 \mu \mathrm{M})$. Recordings were made from organoids silenced with synaptic blockers and TTX. Spatial maps of the median firing rate per channel were generated from organoids treated with synaptic blockers, synaptic blockers in combination with TTX and for the control recordings of untreated organoids. For each treatment, the number of actively spiking channels was counted. This was defined as having at least 30 spikes over the 120 s recording window.

For each channel on the array, the theta oscillation amplitude envelope was computed. ${ }^{69}$ The rms of the theta amplitude envelope was calculated for recordings of the organoids silenced with synaptic blockers and TTX. Subsequently, the fraction of the total recording time that the theta envelope exceeded a threshold of $1,1.5$ or 2 times the rms was computed for each channel, as well as for the burst periods and the non-burst periods of the same channel during the control recording. This fraction of time above the threshold during either the burst period or the nonburst period of the control recording was compared to the blockers and TTX recording. The percent change with respect to the blockers and TTX recording was computed and averaged over all channels that spiked at least 30 times during the control recording. Similarly, signal envelopes were obtained for the delta, theta, alpha, beta and gamma frequency bands. For each frequency 
band in each channel, the rms was computed from the organoids silenced with synaptic blockers and TTX. The fraction of time that the envelope exceeded $2 \mathrm{x}$ the rms was computed for the whole time range of the recording treated with blockers and TTX, as well as for the burst periods of the control recording. The percent change of the fraction of time that the envelope exceeded the threshold was computed for each frequency band in each channel. In addition, the constant decrease in the LFP amplitude over all frequencies after treatment with blockers and TTX was further demonstrated by comparing the power spectral density plots of the same channel during control recordings and during recordings after treatment with blockers and TTX. The LFP power spectrum scaled inversely with frequency $(\sim 1 / f)^{70}$ and was above the electronic noise floor in control conditions (Suppl. Fig 13a-d). The mean theta rms voltage fluctuations measured across all channels for $n=4$ organoids was $7 \pm 2 \mu \mathrm{V}$ (mean \pm STD) for blocker + TTX experiments (Suppl. Fig. 13e), which is in agreement with noise thresholds at this bandwidth for this CMOS architecture in physiological environments. ${ }^{60}$

\section{Theta activity within and outside of neuronal population bursts. Cross-correlation analysis}

of the theta band time series, as described above, was performed on the whole time series and as cross correlations on theta time series occurring within population bursts (detailed in Populationaveraged spike rate and population bursts section of Methods). The number of correlation scores of at least 0.2 was compared between whole theta times series data and the theta time series occurring during population bursts. Next, for each channel that had a correlation score of at least 0.15 with the reference site for the population burst time series, the envelope of the theta filtered LFP was generated and the rms of the envelope was computed. The fraction of the recording for which the envelope exceeded 1.5 times the rms was computed for the recording frames within 
burst periods and the recording frames outside burst periods. The number of channels that showed at least a $10 \%$ increase in the fraction of time during which the envelope was above the rms threshold during bursts periods compared to non-burst periods was counted. Similarly, the number of channels with a $10 \%$ increase during non-burst periods compared to burst periods was counted.

Burst peak-centered theta signal averages. Event triggered averages were made of the theta filtered LFP from each channel using the peak of the population burst as the anchor point. For each channel the theta filtered signal was averaged over all burst events. Spatial maps were generated showing the theta phase per channel at different time points relative to the population burst peak. The amplitude of the envelope of the averaged theta signal was used as a measure for the consistency of the theta phase relative to the population burst peak for multiple burst events. Similarly, the population burst peak centered theta phase was obtained for each burst of spiking activity. Theta phase angle spread was determined by calculating the circular standard deviation of the theta phase relative to the population burst peaks. ${ }^{71}$ The circular standard deviation over all bursts of spiking activity was computed for each time point relative to the burst peak. For each channel where the circular standard deviation fell below 1 within $250 \mathrm{~ms}$ before the burst peak until 500ms after the burst peak, the time point relative to the peak with the lowest circular standard deviation was obtained. Using these relative time points of lowest circular standard deviation, a histogram showed the time relative to the burst peak with the most consistency in the theta phase of the signals taken over all individual spiking activity bursts. 
Spike phase locking to theta. Action potential spike events that occurred at least 30 times were considered for the analysis of each electrode channel. The time series theta phase, $\phi(t)$, was determined by taking the standard Hilbert transformation, $H(t)$, of the theta band oscillation and calculating the angle between the real and imaginary components of $H(t)$ in MATLAB. ${ }^{69}$ The theta phase was then counted at each spike event time for all spiking electrodes. The Rayleigh criterion was used to test the non-uniformity of spikes distributed across the circular phase angles $\left(0^{\circ}, 360^{\circ}\right) .^{39,71,72}$ Spikes were considered to be phase-locked to theta if they passed the Rayleigh criteria test for non-uniformity $(P<0.05)$. To further quantify the ensemble of phase-locked sites identified across the organoids, connectivity edges were identified that formed connections with these nodes. Next, all edges that directly connect phase-locked nodes that were also part of the largest component of the spike connectivity network were used to construct the connectivity ensemble of phase-locked sites across the organoid. The phase-locked ensemble distribution of edge strengths was then compared to the rest of the edges in the largest component of the connectivity network. A truncated power law was fitted to both edge strength distributions for L1 to show the differences between the distributions. These differences were further highlighted by violin plots for $n=3$ organoids.

\section{Data availability}

All data needed to evaluate the conclusions in the paper are present in the paper and/or the Supplementary Materials. Additional data and code related to this paper are available from the corresponding author upon reasonable request. 


\section{References}

1. Del Dosso, A., Urenda, J. P., Nguyen, T. \& Quadrato, G. Upgrading the Physiological Relevance of Human Brain Organoids. Neuron 107, 1014-1028 (2020).

2. Mariani, J. et al. FOXG1-Dependent Dysregulation of GABA/Glutamate Neuron Differentiation in Autism Spectrum Disorders. Cell 162, 375-390 (2015).

3. Muguruma, K., Nishiyama, A., Kawakami, H., Hashimoto, K. \& Sasai, Y. Selforganization of polarized cerebellar tissue in 3D culture of human pluripotent stem cells. Cell Rep. 10, 537-550 (2015).

4. Pasca, A. M. et al. Functional cortical neurons and astrocytes from human pluripotent stem cells in 3D culture. Nat. Methods 12, 671-678 (2015).

5. Quadrato, G. et al. Cell diversity and network dynamics in photosensitive human brain organoids. Nature 545, 48-53 (2017).

6. Qian, X. et al. Brain-Region-Specific Organoids Using Mini-bioreactors for Modeling ZIKV Exposure. Cell 165, 1238-1254 (2016).

7. Birey, F. et al. Assembly of functionally integrated human forebrain spheroids. Nature 545, 54-59 (2017).

8. Lancaster, M. A. et al. Cerebral organoids model human brain development and microcephaly. Nature 501, 373-379 (2013).

9. Sakaguchi, H. et al. Self-Organized Synchronous Calcium Transients in a Cultured Human Neural Network Derived from Cerebral Organoids. Stem Cell Reports 13, 458473 (2019).

10. Giandomenico, S. L. et al. Cerebral organoids at the air-liquid interface generate diverse nerve tracts with functional output. Nat. Neurosci. 22, 669-679 (2019).

11. Qian, X. et al. Sliced Human Cortical Organoids for Modeling Distinct Cortical Layer Formation. Cell Stem Cell 26, 766-781.e9 (2020).

12. Trujillo, C. A. et al. Complex Oscillatory Waves Emerging from Cortical Organoids Model Early Human Brain Network Development. Cell Stem Cell 25, 558-569.e7 (2019).

13. Yuan, X. et al. Versatile live-cell activity analysis platform for characterization of neuronal dynamics at single-cell and network level. Nat. Commun. 11, (2020).

14. Shein-Idelson, M., Pammer, L., Hemberger, M. \& Laurent, G. Large-scale mapping of cortical synaptic projections with extracellular electrode arrays. Nat. Methods 14, 882-890 (2017).

15. Jun, J. J. et al. Fully integrated silicon probes for high-density recording of neural activity. Nature 551, 232-236 (2017).

16. Frey, U. et al. Switch-matrix-based high-density microelectrode array in CMOS technology. IEEE J. Solid-State Circuits 45, 467-482 (2010).

17. Bennett, B. D., Callaway, J. C. \& Wilson, C. J. Intrinsic Membrane Properties Underlying Spontaneous Tonic Firing in Neostriatal Cholinergic Interneurons. J. Neurosci. 20, 84938503 (2000).

18. Study, R. E. \& Barker, J. L. Diazepam and (--)-pentobarbital: fluctuation analysis reveals different mechanisms for potentiation of gamma-aminobutyric acid responses in cultured central neurons. Proc. Natl. Acad. Sci. U. S. A. 78, 7180-7184 (1981).

19. Drexler, B., Zinser, S., Hentschke, H. \& Antkowiak, B. Diazepam decreases action potential firing of neocortical neurons via two distinct mechanisms. Anesth. Analg. 111, 
1394-1399 (2010).

20. Sohal, V. S., Zhang, F., Yizhar, O. \& Deisseroth, K. Parvalbumin neurons and gamma rhythms enhance cortical circuit performance. Nature 459, 698-702 (2009).

21. Hu, H., Gan, J. \& Jonas, P. Fast-spiking, parvalbumin+ GABAergic interneurons: From cellular design to microcircuit function. Science 345, 1255263 (2014).

22. Polioudakis, D. et al. A Single-Cell Transcriptomic Atlas of Human Neocortical Development during Mid-gestation. Neuron 103, 785-801.e8 (2019).

23. Cutts, C. S. \& Eglen, X. S. J. Detecting pairwise correlations in spike trains: An objective comparison of methods and application to the study of retinal waves. J. Neurosci. 34, 14288-14303 (2014).

24. Tovar, K. R. et al. Action potential propagation recorded from single axonal arbors using multielectrode arrays. J. Neurophysiol. 120, 306-320 (2018).

25. Radivojevic, M. et al. Tracking individual action potentials throughout mammalian axonal arbors. Elife 6, 1-23 (2017).

26. Barthó, P. et al. Characterization of neocortical principal cells and interneurons by network interactions and extracellular features. J. Neurophysiol. 92, 600-608 (2004).

27. Fatt, B. P. \& Katz, B. An analysis of the end-plate potential recorded with an intra-cellular electrode. J. Physiol. 320-370 (1951). doi:10.1113/jphysiol.1951.sp004675

28. Miles, R. \& Wong, R. K. S. Latent synaptic pathways revealed after tetanic stimulation in the hippocampus. Nature 329, 724-726 (1987).

29. Sayer, R. J., Friedlander, M. J. \& Redman, S. J. The time course and amplitude of EPSPs evoked at synapses between pairs of CA3/CA1 neurons in the hippocampal slice. $J$. Neurosci. 10, 826-836 (1990).

30. Bassett, D. S., Meyer-Lindenberg, A., Achard, S., Duke, T. \& Bullmore, E. Adaptive reconfiguration of fractal small-world human brain functional networks. Proc. Natl. Acad. Sci. U. S. A. 103, 19518-19523 (2006).

31. Kuśmierz, Ł., Ogawa, S. \& Toyoizumi, T. Edge of Chaos and Avalanches in Neural Networks with Heavy-Tailed Synaptic Weight Distribution. Phys. Rev. Lett. 125, 1-6 (2020).

32. Song, S., Sjöström, P. J., Reigl, M., Nelson, S. \& Chklovskii, D. B. Highly nonrandom features of synaptic connectivity in local cortical circuits. PLoS Biol. 3, 0507-0519 (2005).

33. Lefort, S., Tomm, C., Floyd Sarria, J. C. \& Petersen, C. C. H. The Excitatory Neuronal Network of the C2 Barrel Column in Mouse Primary Somatosensory Cortex. Neuron 61, 301-316 (2009).

34. Buzsáki, G., Anastassiou, C. a \& Koch, C. The origin of extracellular fields and currents-EEG, ECoG, LFP and spikes. Nat. Rev. Neurosci. 13, 407-20 (2012).

35. Nolte, G. et al. Identifying true brain interaction from EEG data using the imaginary part of coherency. Clin. Neurophysiol. 115, 2292-2307 (2004).

36. Petermann, T. et al. Spontaneous cortical activity in awake monkeys composed of neuronal avalanches. Proc. Natl. Acad. Sci. U. S. A. 106, 15921-15926 (2009).

37. Bastien, C. H., LeBlanc, M., Carrier, J. \& Morin, C. M. Sleep EEG power spectra, insomnia, and chronic use of benzodiazepines. Sleep 26, 313-317 (2003).

38. Van Lier, H., Drinkenburg, W. H. I. M., Van Eeten, Y. J. W. \& Coenen, A. M. L. Effects of diazepam and zolpidem on EEG beta frequencies are behavior-specific in rats.

Neuropharmacology 47, 163-174 (2004). 
39. Anastassiou, C. A., Perin, R., Markram, H. \& Koch, C. Ephaptic coupling of cortical neurons. Nat. Neurosci. 14, 217-224 (2011).

40. Mizuseki, K., Sirota, A., Pastalkova, E. \& Buzsáki, G. Theta Oscillations Provide Temporal Windows for Local Circuit Computation in the Entorhinal-Hippocampal Loop. Neuron 64, 267-280 (2009).

41. Jezek, K., Henriksen, E. J., Treves, A., Moser, E. I. \& Moser, M. B. Theta-paced flickering between place-cell maps in the hippocampus. Nature 478, 246-249 (2011).

42. Klausberger T. et al. Brain-state- and cell-type-specific firing of hippocampal interneurons in vivo. Nature 421, 844-848 (2003).

43. Hafting, T., Fyhn, M., Bonnevie, T., Moser, M. B. \& Moser, E. I. Hippocampusindependent phase precession in entorhinal grid cells. Nature 453, 1248-1252 (2008).

44. Amilhon, B. et al. Parvalbumin Interneurons of Hippocampus Tune Population Activity at Theta Frequency. Neuron 86, 1277-1289 (2015).

45. Blankenship, A. G. \& Feller, M. B. Mechanisms underlying spontaneous patterned activity in developing neural circuits. Nat. Rev. Neurosci. 11, 18-29 (2010).

46. LORENTE de NO, R. Analysis of the distribution of the action currents of nerve in volume conductors. Stud. Rockefeller Inst. Med. Res. Repr. (1947).

47. Mitzdorf, U. Current source-density method and application in cat cerebral cortex: Investigation of evoked potentials and EEG phenomena. Physiol. Rev. 65, 37-100 (1985).

48. Einevoll, G. T. et al. Laminar population analysis: Estimating firing rates and evoked synaptic activity from multielectrode recordings in rat barrel cortex. J. Neurophysiol. 97, 2174-2190 (2007).

49. Pettersen, K. H., Hagen, E. \& Einevoll, G. T. Estimation of population firing rates and current source densities from laminar electrode recordings. J. Comput. Neurosci. 24, 291313 (2008).

50. O'keefe, J. \& Nadel, L. Précis of O'Keefe \& Nadel's The hippocampus as a cognitive map. Behav. Brain Sci. 2, 487-494 (1979).

51. Fox, M. D. \& Raichle, M. E. Spontaneous fluctuations in brain activity observed with functional magnetic resonance imaging. Nat. Rev. Neurosci. 8, 700-711 (2007).

52. Rutishauser, U., Ross, I. B., Mamelak, A. N. \& Schuman, E. M. Human memory strength is predicted by theta-frequency phase-locking of single neurons. Nature 464, 903-907 (2010).

53. Karch, C. M. et al. Human fibroblast and stem cell resource from the Dominantly Inherited Alzheimer Network. Alzheimer's Res. Ther. 10, 1-11 (2018).

54. Hernandez, I. et al. A farnesyltransferase inhibitor activates lysosomes and reduces tau pathology in mice with tauopathy. Sci. Transl. Med. 11, 1-18 (2019).

55. Macosko, E. Z. et al. Highly parallel genome-wide expression profiling of individual cells using nanoliter droplets. Cell 161, 1202-1214 (2015).

56. Butler, A., Hoffman, P., Smibert, P., Papalexi, E. \& Satija, R. Integrating single-cell transcriptomic data across different conditions, technologies, and species. Nat. Biotechnol. 36, 411-420 (2018).

57. Stuart, T. et al. Comprehensive Integration of Single-Cell Data. Cell 177, 1888-1902.e21 (2019).

58. McGinnis, C. S., Murrow, L. M. \& Gartner, Z. J. DoubletFinder: Doublet Detection in Single-Cell RNA Sequencing Data Using Artificial Nearest Neighbors. Cell Syst. 8, 329337.e4 (2019). 
59. Potter, S. M. \& DeMarse, T. B. A new approach to neural cell culture for long-term studies. J. Neurosci. Methods 110, 17-24 (2001).

60. Ballini, M. et al. A 1024-channel CMOS microelectrode array with 26,400 electrodes for recording and stimulation of electrogenic cells in vitro. IEEE J. Solid-State Circuits (2014). doi:10.1109/JSSC.2014.2359219

61. Gold, C., Henze, D. A., Koch, C. \& Buzsáki, G. On the Origin of the Extracellular Action Potential Waveform $\square$ : A Modeling Study. J Neurophysiol 95, 3113-3128 (2006).

62. Quiroga, R. Q., Nadasdy, Z. \& Ben-Shaul, Y. Unsupervised Spike Detection and Sorting with Wavelets and Superparamagnetic Clustering. Neural Comput. 16, 1661-1687 (2004).

63. Buzsáki, G., Logothetis, N. \& Singer, W. Scaling brain size, keeping timing: Evolutionary preservation of brain rhythms. Neuron 80, 751-764 (2013).

64. Muthukumaraswamy, S. D. et al. Broadband cortical desynchronization underlies the human psychedelic state. J. Neurosci. 33, 15171-15183 (2013).

65. Hartigan, J. A. \& Hartigan, P. M. The Dip Test of Unimodality. Ann. Stat. 13, 1-16 (2007).

66. Bounova, G. \& De Weck, O. Overview of metrics and their correlation patterns for multiple-metric topology analysis on heterogeneous graph ensembles. Phys. Rev. E - Stat. Nonlinear, Soft Matter Phys. 85, 1-11 (2012).

67. Akaike, H. A New Look at the Statistical Model Identification. IEEE Trans. Automat. Contr. 19, 716-723 (1974).

68. Guggisberg, A. G. et al. Mapping functional connectivity in patients with brain lesions. Ann. Neurol. 63, 193-203 (2008).

69. Tort, A. B. L., Komorowski, R., Eichenbaum, H. \& Kopell, N. Measuring phaseamplitude coupling between neuronal oscillations of different frequencies. $J$. Neurophysiol. 104, 1195-1210 (2010).

70. Voytek, B. \& Knight, R. T. Dynamic network communication as a unifying neural basis for cognition, development, aging, and disease. Biol. Psychiatry 77, 1089-1097 (2015).

71. Berens, P. CircStat: A MATLAB Toolbox for Circular Statistics. J. Stat. Softw. 31, (2009).

72. Kim, H., Ährlund-Richter, S., Wang, X., Deisseroth, K. \& Carlén, M. Prefrontal Parvalbumin Neurons in Control of Attention. Cell 164, 208-218 (2016). 


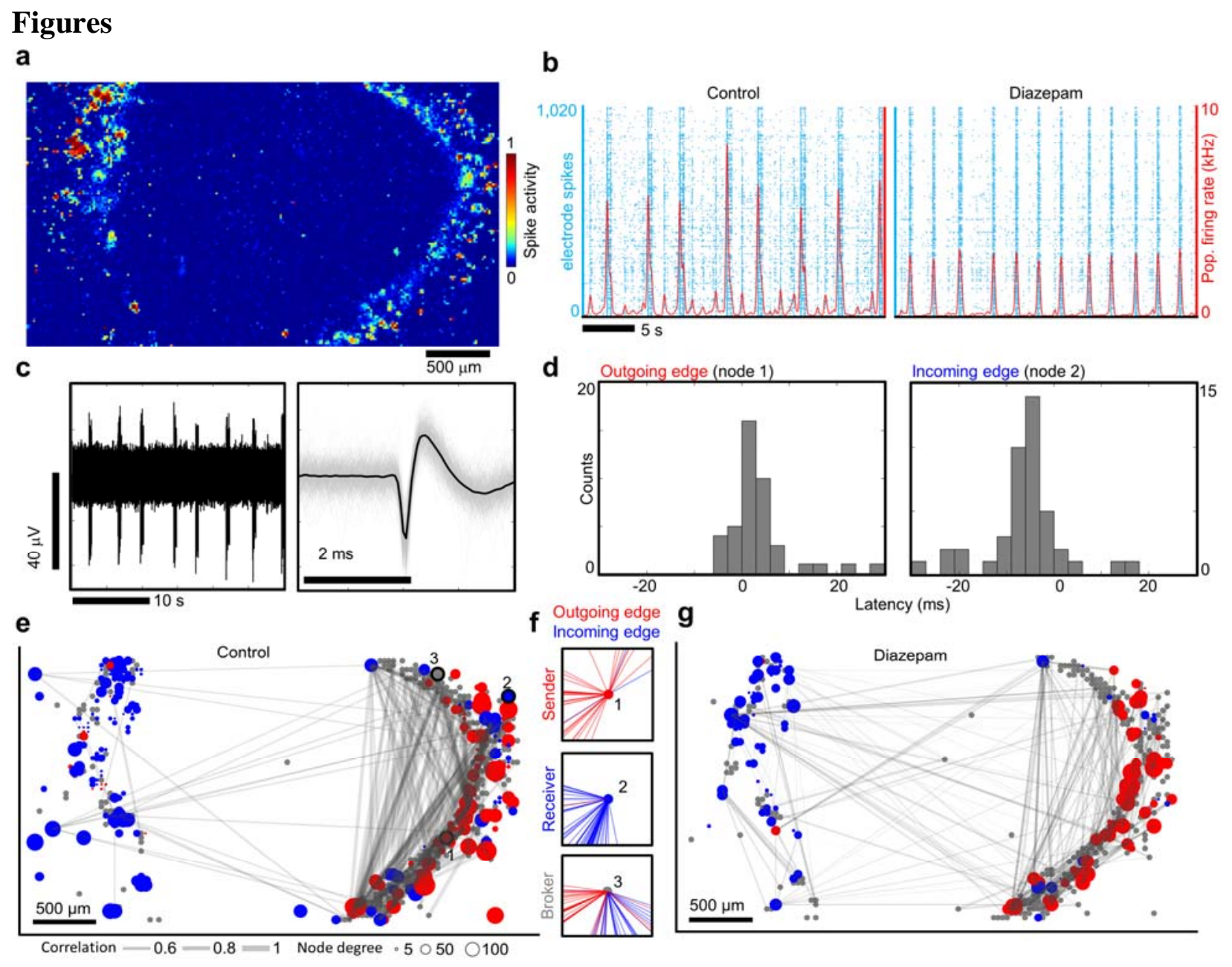

Figure 1. High-resolution maps of extra-cellular action potentials track information flow through the cellular scaffolding of a human cerebral organoid. (a) Spatial map of extra-cellular action potential activity recorded from a human 500 um thick human cerebral organoid slice positioned on a high-density CMOS microelectrode array with 26,400 recording electrodes scanned across 29 contiguous tiled electrode blocks to survey electrical activity across the entire organoid. The color scale indicates the normalized number of detected spikes registered at each electrode for tiled recoding blocks over a $30 \mathrm{~s}$ interval. Scale bar $500 \mu \mathrm{m}$. (b) Left, raster plot of endogenous spiking activity (blue dots) measured from the top active 1,020 recording electrodes. Red line is the average number of spikes detected over a $20 \mathrm{~ms}$ sliding window. Right, same organoid after $50 \mu \mathrm{M}$ diazepam treatment. (c) Left, high-pass filtered data (0.3-7 kHz). Right, spike triggered average extra-cellular action potential waveform (black line) of 565 detected spike instances (gray lines). Notice the stereotyped amplitude distribution of the individual extracellular waveform (gray) compared to the average (black). All channels used for the functional connectivity analysis shown in panels e-g have unimodal amplitude distributions (Hartigans' dip test, $P$ > 0.1 ), corresponding to $94 \%$ of the total electrode channels (see Suppl. Fig. 8 for statistics from $n=6$ organoids). (d) Left, example of the spike time latency distribution between two electrodes (nodes) creating an outgoing edge with a positive mean latency. Right, shows an incoming edge which is characterized by negative mean latency distribution. The time latencies of the spikes indicate the edge directionality between two electrode sites (nodes). (e) Functional connectivity map showing the pairwise correlation strength (edge thickness in gray) between spike trains. Sorted by directionality, the in-degree and out-degree were computed per channel, defined as predominately incoming or outgoing edges 
respectively and designated receiver (blue) nodes, sender (red) nodes. All other nodes were labelled brokers (gray with a fixed size not indicative of node degree). For visual clarity, only the top 150 outgoing and the top 150 incoming edges are shown for sender and receiver nodes, respectively. (f) Examples of single sender (1), receiver (2) and broker (3) nodes showing all incoming (blue) and outgoing (red) edges for the spatial sites identified on the panel e. (g) Functional connectivity map of the same organoid after treatment with $50 \mu \mathrm{M}$ diazepam. The relative number of sender nodes remained unchanged for $n=3$ organoids except for L2 which increased, while the number of receiver nodes decreased $(n=4$, Suppl. Fig. 10).
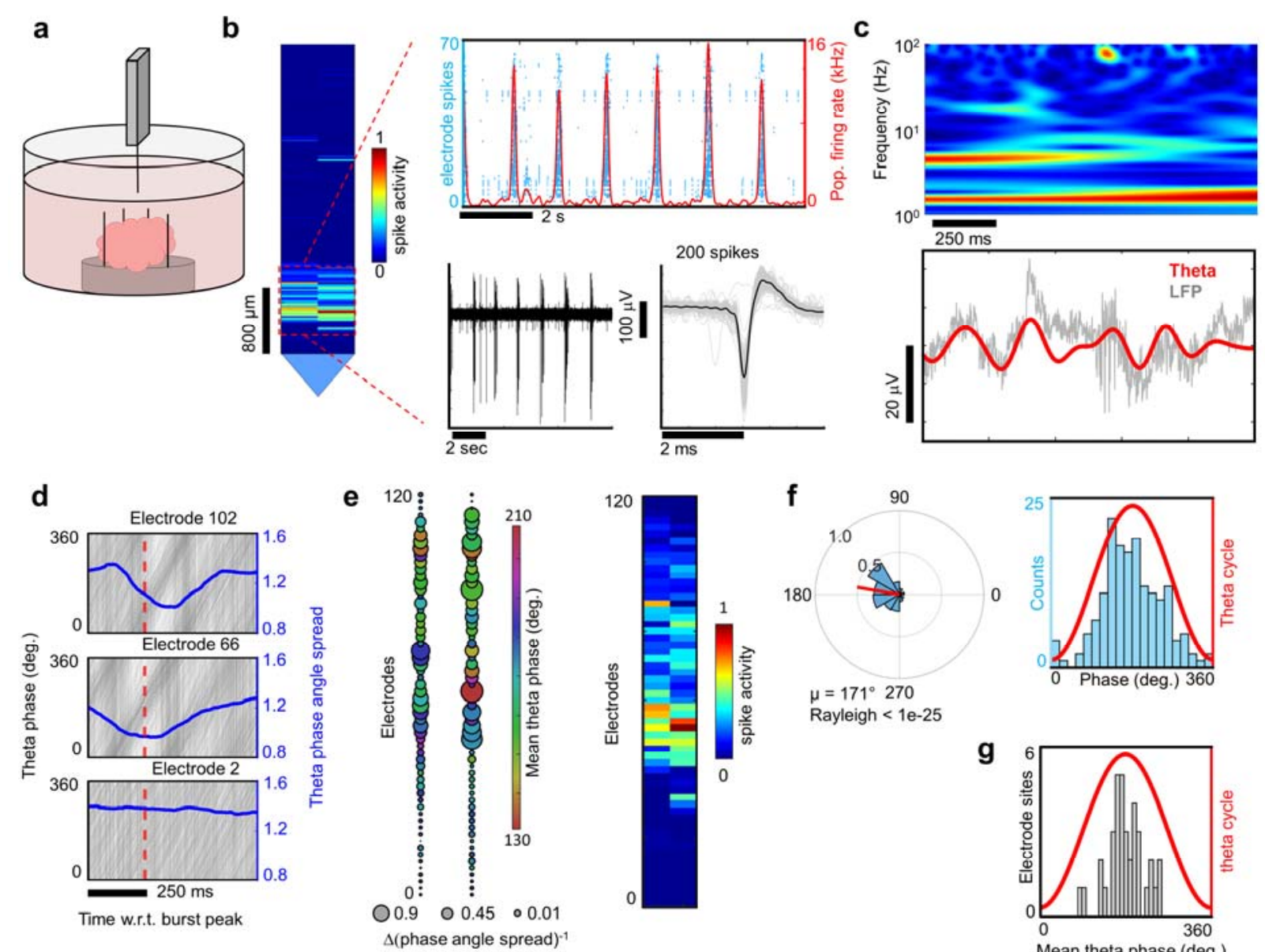

Figure 2. Neuropixels CMOS shanks resolves spiking and LFP in the z-plane. (a) A Neuropixels high-density CMOS shank was attached to a custom-made mount and controlled by a micromanipulator in order to lower the shank into an immobilized cerebral organoid kept at $37{ }^{\circ} \mathrm{C}$ in BrainPhys media. (b) Spikes were detected in a subset of electrodes near the tip of the Neuropixels shank. Among the active electrodes, stereotyped population spiking activity was detected as well as single neuron action potential activity. (c) Top, spectrogram plot of local field potential (LFP) from an electrode illustrates dominant oscillation power in the theta and delta bands. Bottom, raw LFP (gray line) from the same electrode overlaid with the theta-filtered band (red line). (d) Theta phase traces (gray lines) are shown relative to population burst peak events (red dotted line) reveal phase coherence as illustrated by a drop in the phase angle spread (blue line). The bottom plot shows an electrode site displaying no phase coherence relative to population burst events. (e) Left, spatial map of the change in theta phase angle spread is shown relative to burst peak time across the Neuropixels shank. The bubble size indicates the inverse of the phase angle spread relative to the burst peak for each electrode. Notice the overlap with phase coherent 
sites and the spatial region registering spiking activity on the right. (f) Left, circular distribution of thetaspike phase angles measured from a single electrode site. The direction of the mean spike angle $(\mu)$ relative to the theta phase and magnitude (mean resultant length) are shown as polar plots. The Rayleigh criteria for non-uniformity was used to determine if spikes were distributed non-uniformly over the theta cycle $\left(0^{\circ}, 360^{\circ}\right)$. Right, distributions of theta-spike angles shown relative to the theta cycle. (g) Mean theta phase angle of spike phase-locked electrodes across the Neuropixels probe satisfying the Rayleigh criterion $(P<0.05)$.

a
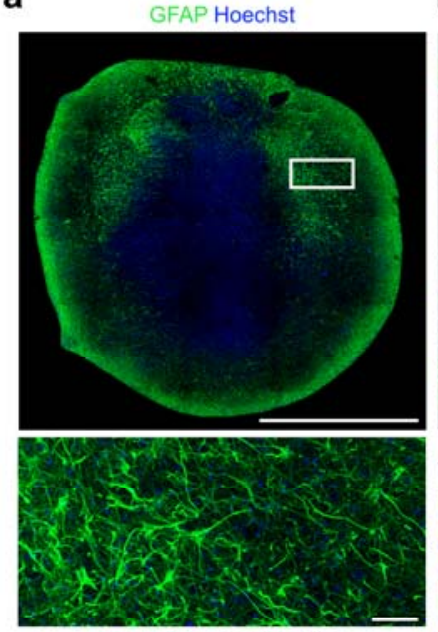

GFAP Connexin Hoechst

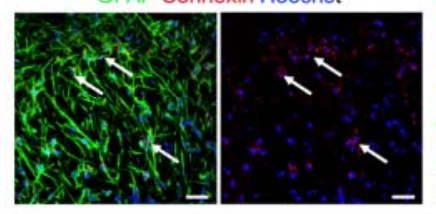

b
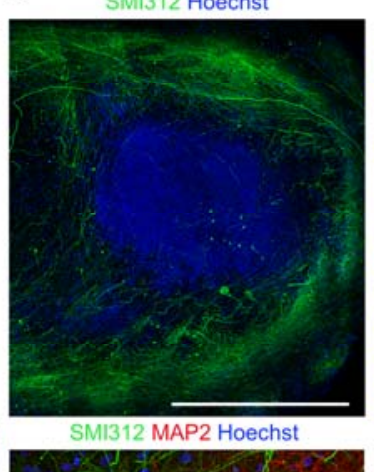

c

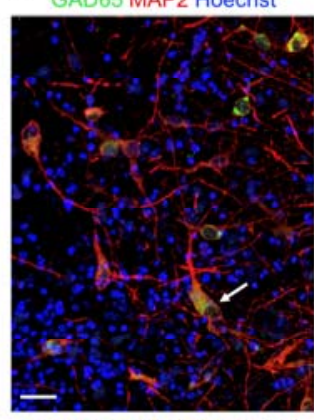

Parvalbumin MAP2 Hoechst

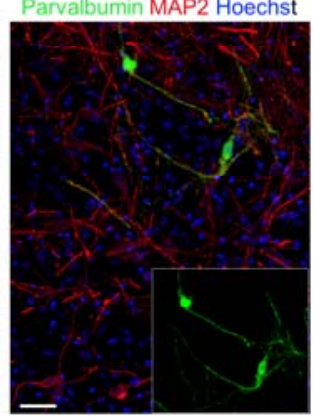

d

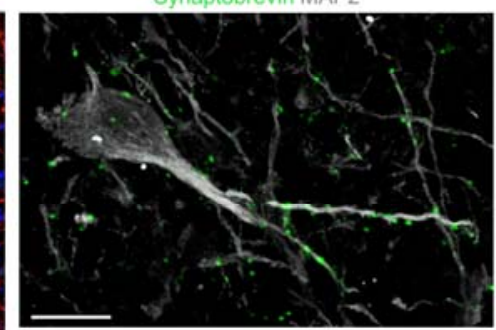

e. GABAergic neurons Astrocytes 1 - Glutamatergic neurons • Astrocytes 2

• Oligodendrocyte progenitors

5 - Choroid plexus

- Cycling cells

- Glia

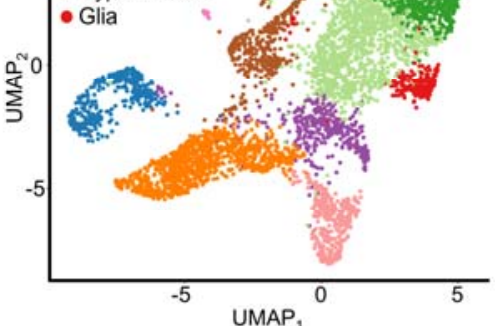

Figure 3. Human cerebral organoids form a scaffolding capable of supporting neuronal microcircuitry. (a) Top, High-resolution, whole-section of an 8-month organoid immunostained with anti-GFAP (green), and counterstained with Hoechst (cell nuclei, blue), scale bar $1 \mathrm{~mm}$. Middle, a high magnification of (top box) showing stellate appearance characteristic of astrocytes. Bottom left, AntiGFAP-positive astrocytes (green) in an 8-month organoid co-labeled with anti-connexin 43 demonstrating gap junctions (red). Bottom right, connexin 43 gap junction (red; arrows). Middle and bottom scale bars are $40 \mu \mathrm{m}$. (b) Top, long neuronal processes labeled with anti-SMI312 (green) in an 8-month organoid, scale bar $500 \mu \mathrm{m}$. Bottom, anti-SMI312 (green) axons neighboring MAP2-positive (red) neurons near the margin of 8-month organoid, scale bar $20 \mu \mathrm{m}$. (c) Top, anti-GAD65 positive neurons (green) co-labeled with anti-MAP2. Bottom, Anti-Parvalbumin-positive neurons (green) in an 8-month organoid co-labeled with anti-MAP2 (red). Scale bars $40 \mu \mathrm{m}$ (d) The pre-synaptic marker synaptobrevin label puncta of a MAP2-positive processes, scale bar $20 \mu \mathrm{m}$. (e) Single-cell RNA sequencing (drop-seq) shows the presence of glutamatergic neurons, GABAergic neurons and astrocyte populations. Single cell transcriptomes (5,680 cells collected from three 7-months-old organoids) are visualized as a Uniform Manifold Approximation and Projection (UMAP). 

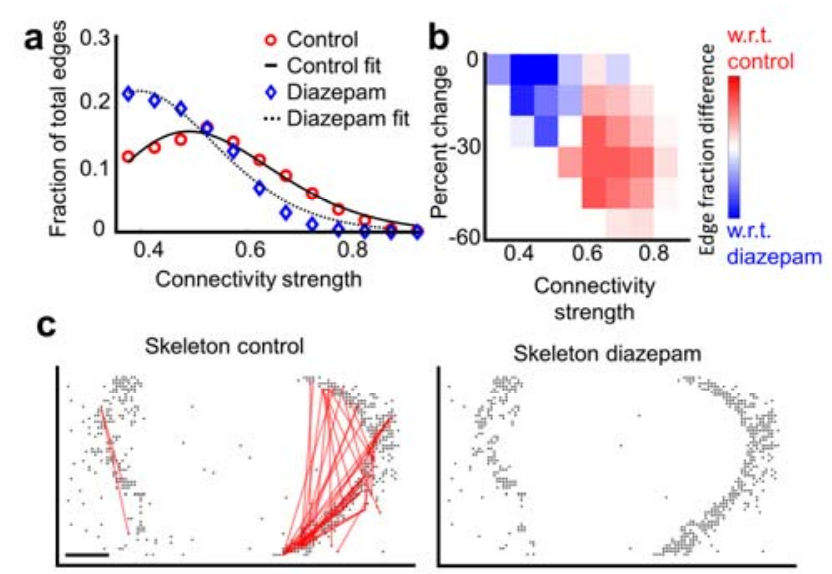

Figure 4. Diazepam selectively modulates the skeleton network of strong connections. (a) Pairwise spike correlation (edge) distributions of the functional connectivity maps shown in Fig. 1e,g. The edge strengths (of the largest component of interconnected nodes) were binned using a bin size $=0.05$ over a connectivity range from 0.35 to 1 . A truncated power law fit illustrates "a skeleton of stronger connections immersed in a sea of weaker ones". Diazepam $(50 \mu \mathrm{M})$ decreases the minority population of stronger connectivity strengths, while increasing the connection strengths of the weaker and more abundant connections with respect to control conditions. (b) For the edges that were present under both control and diazepam conditions, the fraction of edges with high connectivity strength and strong weakening was higher for control whereas the fraction of edges with low connectivity strength and mild weakening was higher for diazepam. (c) Left, connections above a connectivity score of 0.85 are visualized as a skeleton of strong connections. Right, the same top connections are lost with application of $50 \mu \mathrm{M}$ diazepam. The decrease in the skeleton was consistent across $n=3$ organoids (Suppl. Fig. 11g). Scale bar is $500 \mu \mathrm{m}$. 

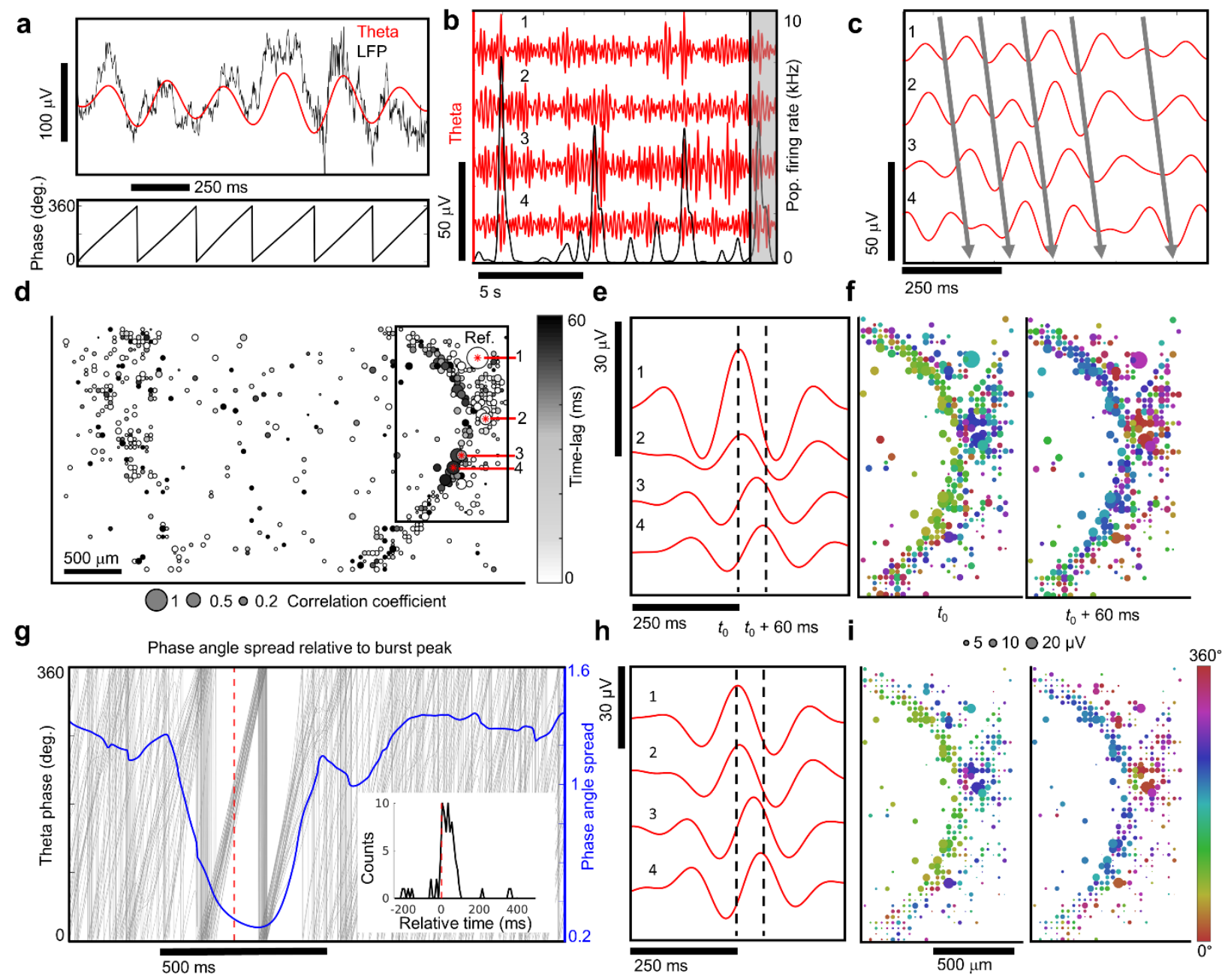

Figure 5. Spatial and temporal coherence of theta oscillations with neuronal population bursts. (a) The raw local field potential $(<500 \mathrm{~Hz}$, black line) and the $4-8 \mathrm{~Hz}$ theta filtered band (red) (top). The phase of the theta oscillation (bottom). (b) Theta band oscillations (red) from four different recording sites and the average population firing rate (black). (c) Zoomed in view of highlighted black rectangle in $\mathbf{b}$. The solid lines indicate the relative phase offsets of theta oscillations across spatial sites of the organoid. (d) Spatial correlation map of theta oscillations. The correlation coefficient (bubble size) is shown with respect to the seed reference site (1) and the relative phase-lag with respect to the reference electrode is shown in grayscale reveal spatial alignment of theta oscillations. (e) Signal averaged theta oscillations using peaks from electrode (1) as a reference. The numbers 1- 4 in c-e,h all refer to the same set of electrodes. (f) Spatial map of signal averaged theta oscillation phase and amplitude relative to reference electrode number 1. Two time points are shown, one at the center of the reference channel $t_{0}$ and another $60 \mathrm{~ms}$ later. (g) Phase angle spread relative to burst peak (blue line). Individual theta phase traces (gray line) are plotted for electrode 1 relative to population burst events. The phase angle spread is minimized after the burst peak (red dotted line). The time of the theta peak amplitude relative to bursts where the angular spread is minimized across multiple electrode sites (inset). (h) The same theta oscillations from e are signal averaged with respect to population burst peak times $\left(t_{0}\right)$. (i) Spatial map of signal averaged theta oscillations using population burst peak times reveal a temporal alignment of theta oscillations with neuronal population bursts. Panels $\mathbf{f}, \mathbf{i}$ share the same scales. 
a

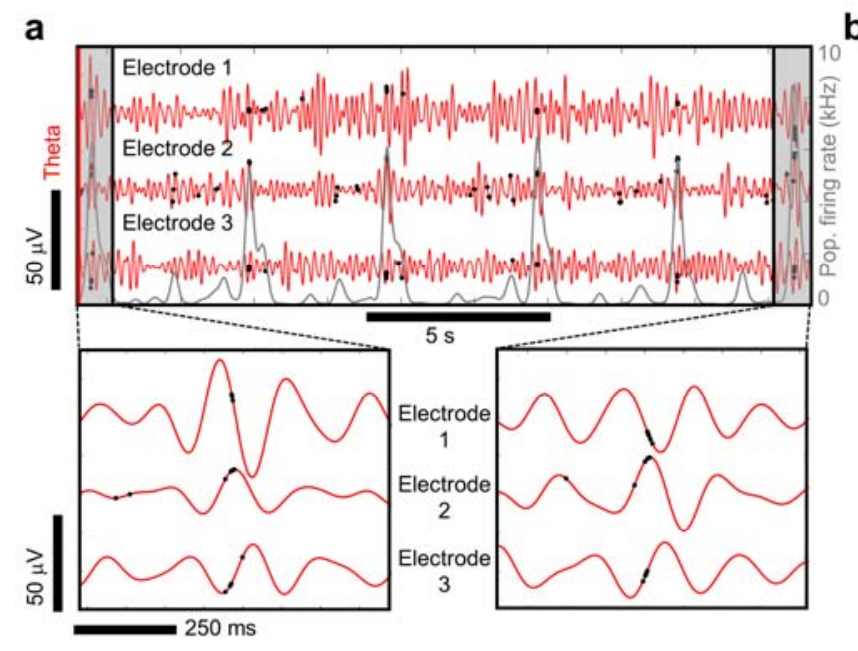

b

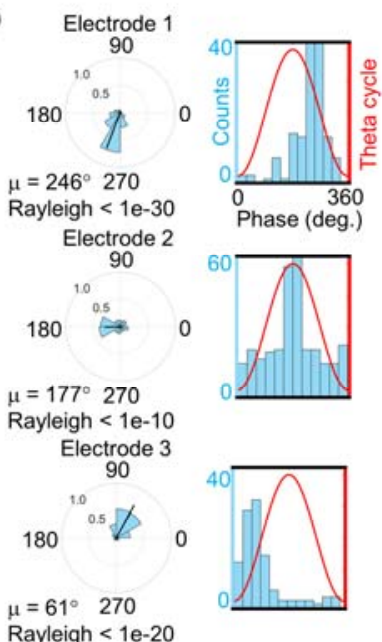

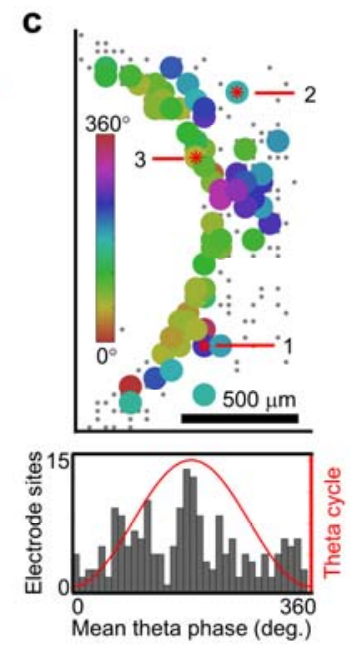

Figure 6. Phase-locking of spikes to theta oscillations. (a) Top, theta oscillations (red line) from three representative electrode sites and the spikes that occurred at each of those electrodes (black dots). The population firing rate across all electrodes (gray line). Bottom, zoomed in view from the gray boxes on the top. (b) Left, circular distribution of theta-spike angles measured from the electrodes in a. Direction of the mean spike angle $(\mu)$ relative to the theta phase and magnitude (mean resultant length) are shown in the polar plots. The Rayleigh criteria for non-uniformity was used to determine if spikes were distributed non-uniformly over the theta cycle $\left(0^{\circ}, 360^{\circ}\right)$. Right, distributions of theta-spike angles shown relative to the theta cycle are visualized as histogram plots. Bottom right, histogram of the mean phased-locked angle to theta for all electrode sites across the array that satisfy the Rayleigh criteria $(P<0.05)$. (c) Top, Cluster of spiking channels phase-locked to theta that overlap with the coherence pocket highlighted by the box in Fig. 5d. The color indicates the mean phase-locked angle to theta $(\mu)$ that satisfy the Rayleigh criteria for non-uniformity $(P<0.05)$. Electrode sites with no preferred theta phase $(P>0.05)$ are shown by gray dots. Bottom, histogram of the mean phased-locked angle to theta for all electrode sites across the array that satisfy the Rayleigh criteria $(P<0.05)$.
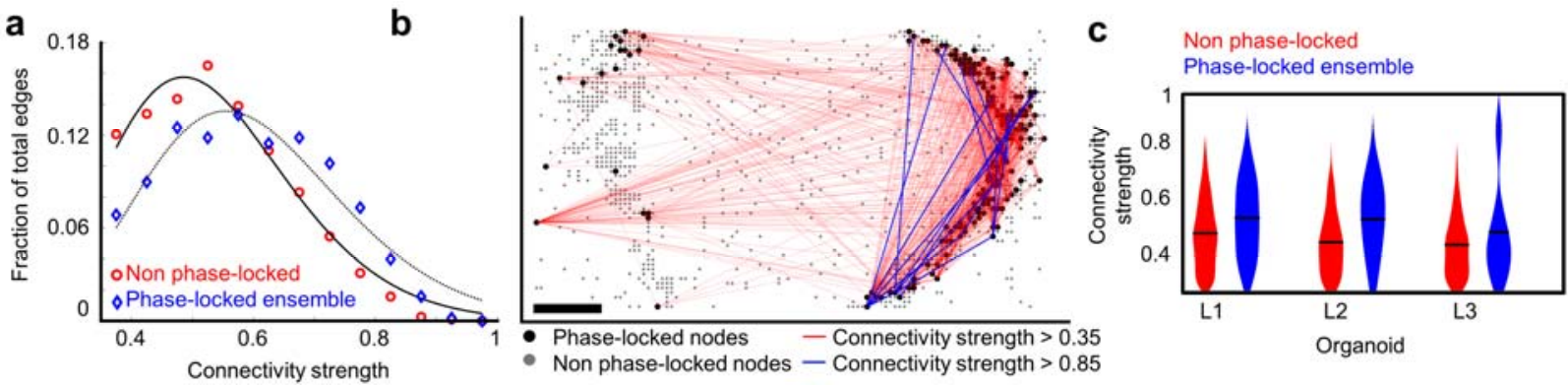

Figure 7. Phase-locked spikes form a strongly interconnected ensemble. (a) The distribution of connectivity (edge) strengths are shown for the largest component (red circles) of interconnected nodes (excluding the interconnected set of phase-locked nodes satisfying the Rayleigh criterion) in organoid L1. On the same graph connectivity strength distributions are shown for the interconnected phase-locked nodes (satisfying the Rayleigh criterion) that constitute a phase-locked ensemble (blue diamonds) with higher connectivity strengths. The lines represent fits to a truncated power law. (b) Spatial map of connectivity strengths among pairwise spiking electrode sites across the organoid that belong to the set phase-locked to theta oscillations (black dots, Rayleigh criterion, $P<0.05$ ). Connectivity strengths from 0.35 to 0.85 are shown as red lines while strengths greater than 0.85 are shown by blue lines. Non-phase locked sites that form the largest interconnected component are shown as gray dots. Scale bar is $500 \mu \mathrm{m}$. 
bioRxiv preprint doi: https://doi.org/10.1101/2021.01.28.428643; this version posted January 28, 2021. The copyright holder for this preprint (which was not certified by peer review) is the author/funder, who has granted bioRxiv a license to display the preprint in perpetuity. It is made available under aCC-BY-NC-ND 4.0 International license.

(c) Connectivity strength distributions (violin plots, $n=3$ organoids) within theta phase-locked ensembles (blue) have a larger fraction of strong connections relative to the non-phase locked network of the largest component of interconnected nodes in which they reside (red), a trend replicated across $n=3$ organoids. 